Revista Brasileira de Cartografia

ISSN 1808-0936 | https://doi.org/10.14393/revbrascartogr

Sociedade Brasileira de Cartografia, Geodésia, Fotogrametria e Sensoriamento Remoto

\title{
Métodos de Classificação e Análise de Trajetórias de Uso e Cobertura da Terra na Amazônia: Implicações para Estudos de Regeneração Florestal
}

\section{Land Use and Land Cover Trajectory Classification and Analysis Methods in the Amazon: Implications for Forest Regeneration Studies}

\author{
Mariane Souza Reis ${ }^{1}$, Maria Isabel Sobral Escada ${ }^{2}$, Sidnei João Siqueira Sant'Anna ${ }^{3}$ e Luciano Vieira Dutra ${ }^{4}$
}

1 Instituto Nacional de Pesquisas Espaciais, PPGCST, São José dos Campos/SP, Brasil. mariane.reis@inpe.br ORCID: https://orcid.org/0000-0001-9356-7652

2 Instituto Nacional de Pesquisas Espaciais, DIOTG, São José dos Campos/SP, Brasil. isabel.escada@inpe.br ORCID: https://orcid.org/0000-0002-5822-8265

3 Instituto Nacional de Pesquisas Espaciais, DIOTG, São José dos Campos/SP, Brasil. sidnei.santanna@ inpe.br ORCID: https://orcid.org/0000-0001-7557-3215

4 Instituto Nacional de Pesquisas Espaciais, DIOTG, São José dos Campos/SP, Brasil. luciano.dutra@inpe.br ORCID: https://orcid.org/0000-0002-7757-039X

Resumo: A análise de processos de regeneração florestal provê informações que permitem estimar serviços ecossistêmicos como a fixação de carbono atmosférico, a recuperação da fertilidade dos solos, a manutenção do ciclo hidrológico e da biodiversidade, entre outros. Esses estudos demandam informações usualmente contidas em trajetórias de uso e cobertura da terra com resolução temporal anual, em intervalos de tempos longos. Comumente, esses dados são obtidos a partir do processamento de séries temporais de imagens de sensoriamento remoto. O objetivo deste artigo de revisão é identificar e descrever os principais métodos utilizados para classificar e analisar trajetórias de uso e cobertura da terra a partir de dados de sensoriamento remoto orbital. Esses métodos foram então discutidos em função de sua aplicabilidade na região Amazônica para análise de regeneração florestal. Observou-se que a análise de trajetórias de uso e cobertura da terra na Amazônia não é trivial. Métodos tradicionais de detecção de mudanças resultam em trajetórias inválidas ou compreendem vários passos de classificação. Pelo grande volume de dados analisados, muitas vezes a informação da trajetória é simplificada ao ponto de se tornar uma análise que envolve apenas um ou dois tempos observados, perdendo-se informações importantes para a análise de regeneração florestal, como a persistência da vegetação secundária ou o tempo de uso antes do abandono, por exemplo. Dentre as principais limitações observadas, destaca-se a baixa disponibilidade de dados, sejam imagens livres de nuvens ou dados de referência.

Palavras-chave: Regeneração florestal. Amazônia. Trajetórias de uso e cobertura da terra. Séries temporais de sensoriamento remoto.

\begin{abstract}
The analysis of forest regeneration processes provides information used to estimate atmospheric carbon assimilation, soil fertility recovery, hydrological cycles and biodiversity maintenance, among other environmental services. These studies require information that is usually found in land use and land cover trajectories mapped in long time intervals and with annual observations. These trajectories are usually obtained by processing remote sensing image time series. In this review article we first identify and describe the main methods used to classify and analyze land use and land cover trajectories based on orbital remote sensing data. We then discuss these methods based on their applicability in forest regeneration studies in the Amazon. Throughout this process we observe that analyzing land use and land cover trajectories in the Amazon is not a trivial task. Traditional change detection methods result in invalid trajectories or require many classification steps. Given the large volume of data, it is common to simplify the information contained within the trajectories to the point that analyses are reduced to one or two observed times. In these cases, important information about regeneration processes is lost, such as persistence of secondary vegetation and time of use before abandonment. Among the main observed limitations, we highlight the lack of available data, such as cloud free images and reference data.
\end{abstract}

Keywords: Forest regeneration. Amazon. Land use and land cover trajectories. Remote sensing time series. 


\section{INTRODUÇÃO}

A Bacia Amazônica é considerada como um dos sistemas ecológicos mais importantes do planeta (FOLEY et al., 2007). Dos mais de 6 milhões de $\mathrm{km}^{2}$ abrangidos por essa bacia, aproximadamente 5,5 milhões de $\mathrm{km}^{2}$ são recobertos por floresta tropical. Esta é a maior floresta tropical do mundo e provê importantes serviços ecossistêmicos como manutenção da biodiversidade, armazenamento de carbono, regulação do fluxo de água e regulação climática regional e global (FOLEY et al., 2007; COE et al., 2013). No Brasil, atribui-se o nome de Amazônia ao bioma amazônico, que ocupa quase metade do território do país e abrange as regiões Norte, Nordeste e Centro-Oeste. Para efeitos legais e de planejamento, instituiu-se também a Amazônia Legal Brasileira que abrange a totalidade dos estados do Acre, Amapá, Amazonas, Mato Grosso, Pará, Rondônia, Roraima e Tocantins, e parte do estado do Maranhão (BRASIL, 1966; BRASIL, 2007).

Apesar de sua importância, a Amazônia tem sofrido constante pressão local e regional sobre seus recursos naturais. Essa pressão, reforçada pelo tipo de desenvolvimento econômico praticado na região e pela atual e ineficiente política de controle, conservação e gestão das florestas, comumente se reflete em mudanças do uso e cobertura da terra, sendo uma das mudanças mais estudadas o desmatamento de florestas primárias (FEARNSIDE, 1990; COE et al., 2013). Dentre os impactos potenciais do desmatamento, podem ser citados: perda da biodiversidade, emissões de gases do efeito estufa, alteração do albedo da superfície, perda da produtividade do solo e alteração de regimes hidrológicos (FEARNSIDE, 2005). Alguns desses impactos podem ser compensados por processos de regeneração florestal, que corresponde ao crescimento de vegetação secundária em áreas cuja cobertura florestal original foi removida (BROWN; LUGO, 1990; CORLETT, 1995). Durante a regeneração florestal se assimila carbono da atmosfera, que é transformado em biomassa ou fixado no solo (RAMANKUTTY et al., 2007). Áreas de vegetação secundária também proveem importantes serviços ambientais como a prevenção da erosão e recuperação da fertilidade do solo, a manutenção do ciclo hidrológico, e a formação e/ou complementação de áreas de corredores ecológicos (CHAZDON et al., 2009; MEYFROIDT; LAMBIN, 2011).

Apesar de se tratar de importante informação para estimativas de emissões de gases do efeito estufa e subsidiar o planejamento territorial (VIEIRA et al., 2014; PARÁ, 2015), detalhes sobre a distribuição espaçotemporal e a intensidade dos processos de regeneração florestal em longos períodos de tempo ainda são pouco conhecidos. A Amazônia é uma região extensa e apresenta históricos de ocupação variados. Assim, os processos de mudanças de uso e cobertura da terra são heterogêneos no espaço e no tempo, o que pode afetar distintamente os processos de regeneração florestal (MORAN et al., 2000; PERZ; SKOLE, 2003; WANDELLI; FEARNSIDE, 2015) e resultar em diferentes dinâmicas de acúmulo de biomassa e taxas de absorção de carbono (STEININGER, 2000; AGUIAR et al., 2012).

Como a regeneração florestal pode envolver diferentes ciclos de exploração, o estudo desse processo demanda informações acerca de trajetórias de uso e cobertura da terra, que são definidas como a sucessão de tipos (i.e. classes) de uso e/ou cobertura da terra para uma dada unidade espacial de análise em três ou mais tempos observados (MERTENS; LAMBIN, 2000; MENA, 2008). Cobertura da terra se refere ao estado biofísico da superfície terrestre, o que engloba a quantidade e o tipo de cobertura vegetal, de água e de outros materiais e estruturas de origem natural ou antrópica. Uso da terra refere-se ao conjunto de atividades exercidas e o intuito da manipulação da superfície terrestre, ou seja, o propósito para o qual a cobertura da terra é utilizada por atividades de interesse humano (TURNER; MEYER, 1994).

Comumente, trajetórias de uso e cobertura da terra são obtidas a partir do processamento de séries temporais de imagens de sensoriamento remoto orbital ${ }^{1}$. Nos últimos anos, avanços computacionais e metodológicos, a disponibilidade sem precedentes de dados de diferentes sensores, além da transparência e padronização dos formatos de apresentação desses dados (WULDER et al., 2018) propiciaram o avanço no

\footnotetext{
${ }^{1}$ Informações sobre o uso da terra não são diretamente deriváveis da análise de dados de sensoriamento remoto. No entanto, o uso pode ser atribuído pelo analista em função do conhecimento da área de estudo, em associação às classes de cobertura e/ou com a utilização de dados auxiliares.
} 
desenvolvimento de novas técnicas de análise de séries temporais de dados de sensoriamento remoto. Algumas dessas técnicas foram revisadas nos estudos de Banskota et al. (2014), Gómez et al. (2016) e Zhu (2017). Por se tratarem de estudos abrangentes e mais gerais, algumas especificidades importantes na análise de trajetórias de regeneração florestal na Amazônia não foram detalhadas nos mesmos. Nesse sentido, o objetivo do presente artigo é identificar e descrever os principais métodos utilizados para classificar e analisar trajetórias de uso e cobertura da terra a partir de dados de sensoriamento remoto orbital. Devido ao elevado grau de complexidade dos processos de regeneração florestal, é importante que estudos relacionados tenham objetivos claramente estabelecidos para a correta definição das classes de uso e cobertura da terra, bem como a determinação apropriada das resoluções e extensões espacial e temporal das análises, uma vez que esses fatores condicionam a escolha de dados e técnicas adequadas. Essas características são abordadas nesse artigo e discutidas em função do potencial e das limitações dos métodos, ferramentas e produtos de sensoriamento remoto disponíveis.

\subsection{Definição adotada e escopo de revisão}

Existem diferentes termos adotados na literatura para a descrição de áreas de vegetação secundária. Os termos mais comuns e variações de definição estão apresentados no Quadro 1. Outras definições foram discutidas por Chokkalingam e Jong (2001).

Quadro 1 - Definições de vegetação secundária encontradas na literatura.

\begin{tabular}{|c|c|}
\hline Termo & Definição/Autores \\
\hline \multirow[t]{3}{*}{ Vegetação secundária } & $\begin{array}{l}\text { Vegetação formada em consequência de impactos antrópicos em áreas de florestas, com ênfase para } \\
\text { aquelas que resultam do abandono de áreas desflorestadas para uso agropecuário, excluindo-se florestas } \\
\text { plantadas e aquelas que resultam de distúrbios naturais (BROWN; LUGO, 1990) }\end{array}$ \\
\hline & $\begin{array}{l}\text { Área onde houve corte raso detectado pelo Programa de Monitoramento do Desmatamento da Floresta } \\
\text { Amazônica Brasileira por Satélite (PRODES) e que, após abandono, houve regeneração da vegetação } \\
\text { (ALMEIDA et al., 2010) }\end{array}$ \\
\hline & $\begin{array}{l}\text { Áreas originalmente de florestas, que sofreram corte raso e foram utilizadas para silvicultura, agricultura ou } \\
\text { pasto e então deixadas para regeneração, retornando à sua aparência florestal (ALMEIDA et al., 2016) }\end{array}$ \\
\hline \multirow[t]{4}{*}{$\begin{array}{l}\text { Floresta } \\
\text { secundária/Floresta de } \\
\text { crescimento } \\
\text { secundário }\end{array}$} & $\begin{array}{l}\text { Florestas se regenerando em grande parte por processos naturais, após distúrbio antrópico e/ou natural } \\
\text { significante da vegetação florestal original em um único ponto no tempo ou sobre um período prolongado, } \\
\text { e que exibem grande diferença na estrutura florestal e/ou na composição de espécies em relação às florestas } \\
\text { primárias próximas ou em locais similares (CHOKKALINGAM; JONG, 2001) }\end{array}$ \\
\hline & $\begin{array}{lcccc}\text { Florestas que se desenvolveram após completo desflorestamento } & \text { (CORLETT, } & \text { 1995; } \\
\text { PUTZ; REDFORD, 2010; VIEIRA et al., 2014; WANG et al., 2020) } & & \\
\end{array}$ \\
\hline & $\begin{array}{l}\text { Vegetação que se desenvolve em áreas cultivadas abandonadas ou em plantações em que a floresta original } \\
\text { foi cortada antes do uso agropecuário (KAMMESHEIDT, 2002) }\end{array}$ \\
\hline & $\begin{array}{l}\text { Aquela que ocorre quando um pixel classificado como alguma cobertura antrópica em um dado ano é } \\
\text { substituído no ano seguinte por um pixel de cobertura florestal que não seja de plantações ou manguezal } \\
\text { (SILVA JUNIOR et al., 2020) }\end{array}$ \\
\hline \multirow[t]{4}{*}{ Capoeira } & Vegetação secundária que nasce após a derrubada das florestas primárias (IBGE, 2004) \\
\hline & $\begin{array}{l}\text { Áreas que, após terem sido radicalmente alteradas por atividades antrópicas, apresentam vegetação } \\
\text { secundária em diferentes estágios de regeneração natural e estão temporária ou permanentemente } \\
\text { removidas do ciclo produtivo agrícola (COSTA, 2016) }\end{array}$ \\
\hline & $\begin{array}{l}\text { Tratos de áreas de variadas dimensões, em estágios diferenciados de regeneração espontânea de cobertura } \\
\text { florestal em ecossistemas alterados de modo radical por ação humana (COSTA, 2009) }\end{array}$ \\
\hline & Vegetação secundária em estágio intermediário de sucessão (SALOMÃO et al., 2012) \\
\hline \multirow[t]{2}{*}{ Capoeirão } & Estágio mais avançado da capoeira no processo de sucessão vegetal (IBGE, 2004) \\
\hline & Vegetação secundária em estágio avançado de sucessão (SALOMÃO et al., 2012) \\
\hline Capoeirinha & $\begin{array}{l}\begin{array}{l}\text { Vegetação secundária em estágio inicial de } \\
\text { (SALOMÃO et al., 2012) }\end{array} \\
\end{array}$ \\
\hline
\end{tabular}

Fonte: Os autores (2020).

As definições apresentadas convergem de alguma forma à ideia de que vegetação secundária, florestas secundárias ou capoeiras são tipos de vegetação que se regeneram após a cobertura florestal original sofrer algum tipo de distúrbio. As principais diferenças observadas entre as definições se referem:

a) ao tipo de distúrbio: se o distúrbio é de origem natural e/ou antrópica ou apenas antrópica;

b) à intensidade do distúrbio: se ocorrem apenas em áreas totalmente desmatadas ou se podem 
ocorrer em áreas com determinados níveis de intensidade de degradação florestal;

c) ao uso atribuído após o distúrbio: algumas definições trazem os tipos de usos que precedem a vegetação secundária;

d) à estrutura e composição da vegetação: se a vegetação resultante deve apresentar características distintas da cobertura original;

e) ao processo de regeneração: se natural ou se ocorre a partir de intervenção humana;

f) ao estágio sucessional: em alguns casos, alguns termos podem se referir a estágios de sucessão distintos, enquanto em outros os mesmos termos são usados de forma generalizada, como é o caso do termo "capoeira";

g) às especificidades dos dados utilizados nos estudos: determinadas definições claramente estão associadas à capacidade de identificação da vegetação secundária por meio de dados de sensoriamento remoto, como as definições de Almeida et al. (2010) e Silva Junior et al. (2020).

Na delimitação de escopo do presente artigo de revisão, o termo vegetação secundária foi reservado para descrever a vegetação não propositadamente plantada que cresce após a completa remoção da cobertura florestal original, sem distinção da causa da remoção dessa cobertura ou tipos de uso intermediários. A regeneração de florestas degradadas não foi incluída nas presentes discussões.

\section{ANÁLISE DE TRAJETÓRIAS DE USO E COBERTURA DA TERRA}

Os métodos e procedimentos para a análise de trajetórias de uso e cobertura da terra a partir de dados de sensoriamento remoto foram reunidos em três seções correspondentes às etapas básicas dos estudos de trajetórias, ilustradas na Figura 1. São essas etapas: 1) a definição do problema; 2) a classificação de trajetórias; e 3) a extração e análise de informações acerca das trajetórias. Essas etapas, bem como suas particularidades em estudos de regeneração florestal, estão descritas a seguir.

Figura 1- Etapas e fatores associados para análises de trajetórias de uso e cobertura da terra baseadas em dados de sensoriamento remoto.

\begin{tabular}{|c|c|c|c|c|c|c|}
\hline Etapas & \multicolumn{6}{|c|}{ Fatores asso } \\
\hline $\begin{array}{c}\text { Definição do } \\
\text { problema }\end{array}$ & $\begin{array}{l}\text { Área de estudo } \\
\text { •Localização } \\
\text { •Extensão } \\
\text { •Complexidade }\end{array}$ & \multicolumn{2}{|c|}{$\begin{array}{l}\text { Processos de interesse } \\
\text {-Período de análise } \\
\text { •Definição de classes de uso e } \\
\text { cobertura da terra }\end{array}$} & \multicolumn{3}{|c|}{$\begin{array}{l}\text { Seleção de dados de entrada } \\
\text { •Resoluções } \\
\text {-Disponibilidade } \\
\text { •Condições ambientais e de imageamento }\end{array}$} \\
\hline $\begin{array}{l}\text { Classificação de } \\
\text { trajetórias }\end{array}$ & \multicolumn{2}{|c|}{$\begin{array}{l}\text { Pré-processamento } \\
\text { •Correções geométricas e } \\
\text { radiométricas } \\
\text { •Remoção de áreas sem informação } \\
\text { •Composição de imagens } \\
\text { •Extração e seleção de variáveis }\end{array}$} & \multicolumn{3}{|c|}{$\begin{array}{l}\text { Métodos de classificação } \\
\text {-Número de variáveis } \\
\text { - Tamanho da série temporal } \\
\text {-Correção de trajetórias } \\
\text { inválidas } \\
\text { - Dados multissensores }\end{array}$} & $\begin{array}{l}\text { Análise da qualidade } \\
\text { •Índices de exatidão } \\
\text { •Análise da validade das } \\
\text { transições } \\
\text { •Análises de incerteza }\end{array}$ \\
\hline $\begin{array}{l}\text { Extração e análise de } \\
\text { informações }\end{array}$ & \multicolumn{2}{|c|}{$\begin{array}{l}\text { Análises mono e bitemporal } \\
\text { •Área e/ou padrão de classes/data } \\
\text { •Data/frequência de transições } \\
\text {-Área, tipo e padrão das transições }\end{array}$} & \multicolumn{2}{|c|}{$\begin{array}{l}\text { Simplificação de } \\
\text { trajetórias } \\
\text { •Definição de tipologia } \\
\text { •Algoritmos de agrupamento }\end{array}$} & & $\begin{array}{l}\text { Inálise de processos } \\
\text { ração/intensidade de } \\
\text { essos } \\
\text { acterização de trajetórias }\end{array}$ \\
\hline
\end{tabular}

Fonte: Os autores (2020).

\subsection{Definição do problema}

Esta etapa envolve a definição dos objetivos do estudo, localização e extensão da área estudada, período analisado, classes de interesse e o nível de exatidão exigido (LU; LI; MORAN, 2014). A partir dessa etapa são definidos os dados e métodos utilizados na análise. No entanto, a disponibilidade de dados de sensoriamento remoto, bem como a existência de métodos capazes de processar e extrair diferentes tipos de 
informação, muitas vezes pode limitar e/ou moldar os objetivos do estudo. Dentre as principais limitações, são destacadas aquelas relacionadas com a resolução espacial e temporal do dado utilizado, sua capacidade de discriminação entre diferentes classes de uso e cobertura da terra, e disponibilidade de séries temporais longas.

\subsection{1 ÁREA DE ESTUDO E PROCESSOS DE INTERESSE}

Os processos de regeneração florestal na Amazônia ocorrem em contextos ecológicos, sociais e políticos distintos (VIEIRA et al., 2014; CHAZDON et al., 2020). Estudos relacionados devem considerar que a vegetação secundária pode estar associada a diferentes sistemas de manejo da terra, com ciclos com extensões temporais variadas. Segundo Costa (2016), a presença de vegetação secundária na Amazônia brasileira pode ser associada a três tipos de decisão acerca do uso da terra: 1) adoção de técnicas que pressupõem áreas de pousio para restauração da fertilidade do solo, como é o caso da agricultura itinerante realizada por populações ribeirinhas e tradicionais; 2) abandono de áreas degradadas e/ou improdutivas, em que foram esgotadas as condições edafo-climáticas para produção; e 3) mudanças nas técnicas utilizadas que levam à intensificação do uso da terra e restrição da produção em áreas menores, de forma que a vegetação se regenera no excedente de terras. Nesse último caso, o excedente de terras pode ser novamente incorporado ao processo produtivo, o que resulta no desmatamento da vegetação secundária. Mello e Alves (2011) mencionam ainda um tipo de vegetação secundária que pode decorrer também do abandono de uma área recentemente desmatada, que nunca fez parte do processo de produção. Essas características influenciam na definição do período de análise e das classes de uso e cobertura.

Segundo Wang et al. (2020), áreas de vegetação secundária são comumente desmatadas em até 2 anos após o início da regeneração. Com base nessas informações, conclui-se que a regeneração florestal pode envolver mudanças abruptas ou sutis, de curto ou longo prazo (NUNES et al., 2020). Regiões ribeirinhas, por exemplo, podem apresentar atividades agrícolas com períodos de pousio e regeneração que variam entre menos de quatro ou mais de 15 anos (STEININGER, 2000; AFFONSO et al., 2016; JAKOVAC et al., 2017). As trajetórias de uso e cobertura da terra também podem ser utilizadas para estimar a idade da vegetação secundária (NUNES et al., 2020; SILVA JUNIOR et al., 2020). No estado do Pará, a Instrução Normativa 08 de 28 de outubro de 2015 apresenta regras distintas para a supressão de vegetação secundária em função da idade da mesma. Essas regras são estratificadas em: áreas de vegetação secundária com menos de cinco anos, entre cinco e 20 anos, e com mais de 20 anos (PARÁ, 2015). A diferenciação entre áreas de vegetação secundária com menos e mais de 20 anos também é utilizada para estimativa de taxas de absorção de carbono (SILVA JUNIOR et al., 2020). Dessa forma, recomenda-se a utilização de mapeamentos de uso e cobertura da terra com pelo menos uma observação por ano para o estudo de regeneração florestal na Amazônia, de forma a melhorar a precisão das estimativas de idade e possibilitar a captura de mudanças abruptas em áreas dinâmicas; e em intervalos de tempo superiores a 20 anos, para a observação de completa de ciclos de regeneração mais longos e/ou estimativas de idade.

A definição das classes de uso e cobertura da terra é uma etapa particularmente importante para estudos de trajetórias de regeneração florestal, porque determinadas definições de classes podem resultar em análises bastante distintas. Por exemplo, a classe "floresta" por vezes engloba áreas de floresta primária, de floresta degradada, e também de vegetação secundária, o que não é compatível com estudos de regeneração florestal (PUTZ; REDFORD, 2010). Também é de particular interesse a distinção entre as classes de agricultura, pastagem, áreas urbanas e áreas de mineração, de forma a melhor entender a influência de diferentes históricos de uso. Há três formas básicas de definir classes de uso e cobertura da terra. A primeira delas é simplesmente definir quais são as classes necessárias para o objetivo do estudo, de forma arbitrária. A segunda é derivar classes de um sistema de classificação ou legenda padrão. Um sistema de classificação é um arcabouço lógico que retém os nomes das classes, o critério de separação entre classes e o relacionamento entre elas (MCCONNELL; MORAN, 2001). Uma legenda se trata de um subconjunto de classes, que pode ou não ser obtido de um sistema de classificação (MCCONNELL; MORAN, 2001; HEROLD et al., 2006). Dentre os sistemas de classificação comumente utilizados podem ser citados o Sistema Básico de Classificação da Cobertura e do Uso da Terra (IBGE, 2013), Anderson et al. (1976), CORINE (COOrdination of INformation on the Environment) Land Cover (CLC) (EEA, 2019), entre outros. A terceira forma de definir as classes a serem utilizadas vem da análise empírica dos próprios dados e métodos de classificação utilizados 
(PANTALEÃO; DUTRA; SANDRI, 2012; PEREIRA et al., 2018). As principais vantagens e desvantagens de cada método foram discutidas em Reis et al. (2018). Salienta-se, no entanto, que para os três casos, classes definidas utilizando critérios subjetivos podem comprometer tanto a reprodutibilidade de um estudo quanto a comparação de resultados com outros estudos. Uma solução para diminuir a subjetividade na definição das classes é descrevê-las com base em elementos quantificáveis da cobertura terrestre, como proposto pelo Land Cover Meta Language (LCML), inicialmente denominado Land Cover Classification System (LCCS) (DI GREGORIO; JANSEN, 2005). Trata-se de um sistema flexível, utilizado para padronizar a terminologia utilizada para a descrição de classes, e não as classes em si, e tem sido utilizado para descrever as legendas de diferentes localidades, incluindo a Amazônia (COUTINHO et al., 2013; REIS et al.; 2018). Como estudos de trajetórias de uso e cobertura da terra necessariamente implicam no mapeamento dessas classes ao longo do tempo, também é fundamental que haja correspondência entre as classes das legendas utilizadas em cada data.

\subsubsection{SELEÇÃO DE DADOS DE ENTRADA}

A seleção dos dados geralmente ocorre em função das características de resolução do sensor, adequadas para a identificação das classes de uso e cobertura da terra na escala de interesse. As características dos principais sensores remotos com dados gratuitamente disponíveis estão compiladas no Quadro 2.

Quadro 2 - Características das imagens dos principais acervos gratuitos de dados de sensoriamento remoto.

\begin{tabular}{|c|c|c|c|c|c|c|}
\hline \multirow[b]{2}{*}{ Satélite/Sensor } & \multicolumn{4}{|c|}{ Resolução } & \multirow{2}{*}{$\begin{array}{c}\text { Faixa } \\
\text { imageada } \\
(\mathbf{k m})\end{array}$} & \multirow{2}{*}{$\begin{array}{c}\text { Período } \\
\text { de operação }\end{array}$} \\
\hline & $\begin{array}{c}\text { Espacial } \\
(\mathbf{m})\end{array}$ & $\begin{array}{c}\text { Temporal } \\
\text { (dias) }\end{array}$ & $\begin{array}{c}\text { Espectral } \\
(\mu \mathrm{m})\end{array}$ & $\begin{array}{c}\text { Radiométrica } \\
\text { (bits) }\end{array}$ & & \\
\hline NOAA(6-19)/AVHRR(2-3) & 1100 & $<1$ & 4-6 bandas $(0,58$ a 12,50$)$ & 10 & 2400 & 1979 a 2019 \\
\hline Landsat(1-5)/MSS & $68 \times 83$ & $16-18$ & 4 bandas $(0,50 \text { a } 1,10)^{a}$ & $6-8$ & 185 & 1972 a 1999 \\
\hline Landsat(4-5)/TM & $30^{c}$ & 16 & 7 bandas $(0,45$ a 12,50$)$ & 8 & 185 & 1982 a $2013^{b}$ \\
\hline Landsat-7/ETM+ & $30^{\mathrm{c}, \mathrm{d}}$ & 16 & 8 bandas $(0,45$ a 12,50$)$ & 8 & 183 & a partir de $1999^{\mathrm{e}}$ \\
\hline Landsat-8/OLI & $30^{\mathrm{e}}$ & 16 & 9 bandas $(0,43$ a 2,30$)$ & 12 & 185 & a partir de 2013 \\
\hline Landsat-8/TIRS & 100 & 16 & 2 bandas $(10,30$ a 12,50$)$ & 12 & 185 & a partir de 2013 \\
\hline (Terra/Aqua)/MODIS & $250-1000$ & $1-2$ & 36 bandas $(0,40$ a 14,38$)$ & 12 & 2330 & a partir 1999 \\
\hline Terra/ASTER & $15-90$ & 16 & 15 bandas $(0,52$ a 11,65$)$ & $8-12$ & 60 & a partir de $1999^{\mathrm{f}}$ \\
\hline CBERS(1-2b)/CCD & 20 & 26 & 5 bandas $(0,51$ a 0,89$)$ & 8 & 113 & 1999 a 2010 \\
\hline CBERS(1-2b)/WFI & 260 & $<5$ & 2 bandas $(0,63$ a 0,89$)$ & 8 & 890 & 1999 a 2010 \\
\hline CBERS(1-2)/IRMSS & $80-160$ & 26 & 4 bandas $(0,5$ a 12,50$)$ & 8 & 120 & 1999 a 2008 \\
\hline CBERS-2b/HRC & 2,7 & 130 & 1 banda $(0,50$ a 0,80$)$ & 8 & 27 & 2007 a 2010 \\
\hline CBERS-4/PAN & $5-10$ & $26-52$ & 4 bandas $(0,51$ a 0,89$)$ & 8 & 60 & a partir de 2014 \\
\hline CBERS-4/IRS & $40-80$ & 26 & 4 bandas $(0,50$ a 12,50$)$ & 8 & 120 & a partir de 2014 \\
\hline CBERS-(4-04A)/WFI & $55-64$ & $<5$ & 4 bandas $(0,45$ a 0,89$)$ & 10 & $684-866$ & a partir de 2014 \\
\hline CBERS(4-04A)/MUX & $16,5-20$ & $26-31$ & 4 bandas $(0,45$ a 0,89$)$ & 8 & $95-120$ & a partir de 2014 \\
\hline CBERS-04A/WPM & $2-8$ & 31 & 5 bandas $(0,45$ a 0,90$)$ & 10 & 92 & a partir de 2020 \\
\hline Resourcesat(1-2)/LISS III & 23,5 & 24 & 4 bandas $(0,52$ a 1,70$)$ & 7 & 141 & a partir de 2004 \\
\hline Resourcesat(1-2)/AWiFS & 56 & 5 & 4 bandas $(0,52$ a 1,70$)$ & 10 & $370-810$ & a partir de 2004 \\
\hline Sentinel-2/MSI & $10-60$ & 5 & 13 bandas $(0,43$ a 2,32$)$ & 12 & 290 & a partir de 2015 \\
\hline ALOS/PALSAR & $10-100^{g}$ & s.d. & banda $\mathrm{L}(23 \mathrm{~cm})^{\mathrm{h}}$ & 32 & $30-350^{\mathrm{g}}$ & 2006 a 2011 \\
\hline Sentinel-1 & $5-40^{g}$ & $6-12$ & banda $\mathrm{C}(5,5 \mathrm{~cm})^{\mathrm{i}}$ & 10 & $20-410^{g}$ & a partir de 2014 \\
\hline
\end{tabular}

${ }^{a}$ Sensor carregava uma banda termal no Landsat3. ${ }^{\mathrm{b}}$ Diminuição significativa no número de observações após 2011. ${ }^{\mathrm{c}} 120 \mathrm{~m}$ na banda termal. ${ }^{\mathrm{d}} 15 \mathrm{~m}$ na banda pancromática. ${ }^{\mathrm{e}}$ Imageamento com defeito a partir de $05 / 2003$. ${ }^{\mathrm{f}}$ Saturação nas bandas do infravermelho a partir de 04/2008. ${ }^{\mathrm{g}}$ Dependendo do modo de imageamento. ${ }^{\mathrm{h}} 1$ a 4 polarizações, dependendo do modo de imageamento. ${ }^{\mathrm{i}} 1$ a 2 polarizações, dependendo do modo de imageamento.

Fonte: EMBRAPA (2018), ASF (2020), ESA (2020), INPE (2020a), INPE (2020b), NASA (2020a), NASA (2020b) e USGS (2020). 
Salienta-se que o período de operação de um satélite/sensor não garante a disponibilidade de dados para todas as áreas de estudo. Isso ocorre porque os sensores podem não operar com regularidade em algumas áreas (e.g. o Multispectral Scanner (MSS), com imagens disponíveis para Amazônia em poucas datas). Além disso, sensores remotos ópticos são fortemente influenciados por condições atmosféricas. Como a cobertura de nuvens é frequente na Amazônia em determinadas épocas do ano, é possível que mesmo que uma imagem óptica orbital tenha sido adquirida no período e área desejados, essa não seja apropriada para a análise. Apesar da eventual dificuldade de encontrar imagens livres de nuvens para determinadas datas, dados da família Landsat apresentam características apropriadas para análises de trajetórias anuais de uso e cobertura da terra para longos períodos. Segundo Gómez et al. (2016), imagens Landsat são a base para classificações de cobertura da terra, enquanto os demais dados são utilizados de forma complementar. Ressalta-se, no entanto, o grande potencial de análises futuras serem baseadas em dados Sentinel-2, dada a grande disponibilidade de dados gratuitos, características de resolução, calibração cruzada com dados Landsat, e perspectivas de continuidade do projeto (WULDER et al., 2018).

Dados de Radares de Abertura Sintética (Synthetic Aperture Radar- SAR) são obtidos de forma quase que independente das condições atmosféricas e captam informações da superfície da terra mesmo em áreas cobertas por nuvens (PARADELLA et al., 2005). No entanto, esse tipo de dado é mais difícil de interpretar e processar que os dados ópticos. Eles também estão livremente disponíveis para curtos períodos de tempo. Nesse sentido, apesar da emergência dos primeiros produtos globais de análises florestais baseadas em dados SAR (SHIMADA et al., 2014), análises baseadas unicamente nesse tipo de dado ainda se limitam majoritariamente a poucas datas. Com a continuidade do projeto Sentinel-1, é possível que novos produtos baseados em dados SAR, ou ainda em abordagens multissensores, surjam no futuro.

\subsection{Classificação de trajetórias}

A etapa de classificação de trajetórias corresponde à atribuição de uma classe de uso e cobertura da terra para cada unidade espacial de análise em cada tempo de interesse. Essa etapa envolve três procedimentos principais, descritos nessa seção.

\subsubsection{PRÉ-PROCESSAMENTO}

Uma vez selecionados os dados de sensoriamento remoto a serem utilizados, deve-se garantir que esses sejam de fato comparáveis entre si, no nível da unidade de análise, que geralmente é o próprio pixel da imagem. Essa etapa engloba, basicamente, correções radiométricas e geométricas, que podem ser relativas ou absolutas (COPPIN et al., 2004; RICHARDS; JIA, 2006; WULDER et al. 2018). Também é usual que pixels da imagem tidos como de baixa qualidade ou sem informação sejam mascarados e/ou removidos da análise. Uma forma de solucionar problemas de cobertura de nuvens e/ou falhas de detecção é compor uma nova imagem a partir da seleção de pixels de imagens radiometricamente calibradas do período de interesse. A seleção do melhor pixel disponível (Best Available Pixel - BAP) pode ser feita em função da combinação de diferentes fatores, como baixa probabilidade de nuvens/sombra de nuvens, distância de nuvens, proximidade de data de interesse, índices de qualidade deriváveis das próprias imagens, entre outros, sendo que diferentes técnicas têm sido propostas nos últimos anos (HOLBEN, 1986; GRIFFITHS et al., 2013; WHITE et al., 2014).

Diferentes medidas calculadas a partir dessas imagens podem ser utilizadas para a análise. Além dos próprios valores digitais dos pixels, pode-se utilizar, por exemplo, diferentes tipos de índices derivados da combinação matemática dos canais da imagem, frações subpixel (e.g. frações vegetação, solo e sombra obtidas a partir do Modelo Linear de Mistura Espectral proposto por Shimabukuro e Smith (1995)), medidas de textura, imagens transformadas (e.g. componentes principais, Tasseled Cap) ou imagens fusionadas. Há também métricas específicas que consideram as características multitemporais dos dados (FRANKLIN et al., 2015), ou ainda métricas estatísticas como medianas, quartis e desvio padrão de valores de variáveis derivadas de conjunto de imagens para determinado período (RUFIN et al., 2015; SOUZA et al., 2020). A seleção de uma determinada variável baseia-se (LU et al., 2004): 1) na capacidade de discriminação das classes de uso e cobertura de interesse, 2) na diminuição da dimensionalidade dos 
dados, seja para melhorar a eficiência dos processos de classificação e/ou para evitar a inclusão de ruídos na análise; 3) limitações do método de classificação utilizado, no que se refere à capacidade de utilizar mais de uma variável e/ou data simultaneamente; e 4) necessidade de padronização de dados de sensores distintos e/ou diminuição de interferência de fatores como iluminação e topografia, não resolvidos na calibração radiométrica dos dados.

Dados dos sensores Thematic Mapper (TM), Enhanced Thematic Mapper Plus (ETM+), Operational Land Imager (OLI) e Multispectral Instrument (MSI), respectivamente a bordos dos satélites Landsat 4-5, Landat 7, Landsat 8 e Sentinel 2, são disponibilizados geométrica e radiometricamente corrigidos em diversas plataformas, como por exemplo os repositórios de dados do Serviço Geológico dos Estados Unidos (https://earthexplorer.usgs.gov/, https://glovis.usgs.gov/app). Esses dados corrigidos e com máscara de nuvens também podem ser encontrados no Google Earth Engine (GEE) (GORELICK et al., 2017). Diversos outros algoritmos próprios para o pré-processamento dessas imagens estão discutidos em Zhu (2017), Frantz (2019), e Sanchez et al. (2020).

\subsubsection{MÉTODOS DE CLASSIFICAÇÃO}

A forma mais comum de classificar trajetórias de uso e cobertura da terra é a comparação pósclassificação. Esse método consiste em classificar as imagens de cada data e empilhar essas classificações para formar uma trajetória. Como a classificação é feita de forma independente para cada imagem, geralmente não há necessidade de uma etapa de correção radiométrica, sendo possível utilizar dados multissensores (LU et al., 2004). É necessário o conhecimento acerca da cobertura da terra em cada data, porém não há necessidade de conhecimento prévio das trajetórias de uso e cobertura da terra a serem mapeadas. Uma das principais críticas a esse método reside no fato de que a qualidade da classificação das trajetórias de uso e cobertura da terra depende diretamente dos resultados da classificação em cada data (FULLER; SMITH; DEVEREUX, 2003; TEWKESBURY et al., 2015). Erros de classificação podem resultar em trajetórias inválidas, ou seja, trajetórias de uso e cobertura da terra que apresentam transições inconsistentes (AZEREDO et al., 2016; REIS et al., 2020).

Pode-se também analisar diretamente uma série temporal de imagens radiometricamente calibradas para detectar onde e em que momento houve mudanças na cobertura da terra e, em seguida, classificar esses segmentos temporais de não mudanças em classes de uso e cobertura da terra. Um exemplo de algoritmo que opera dessa forma é o Continuous Change Detection and Classification (CCDC), proposto por Zhu e Woodcock (2014). O CCDC é mais indicado para análises com séries temporais densas (com várias observações por intervalo de interesse), o que não é o caso de análises históricas na Amazônia, em que são encontradas poucas imagens livres de nuvens por ano, sendo que para alguns pontos pode haver apenas uma observação adequada por longos períodos de tempo. Alguns algoritmos desenvolvidos para segmentação no tempo de séries temporais de imagens de satélite estão atualmente disponíveis no GEE (https://developers.google.com/earth-engine/api_docs). Dentre eles, o Landsat-based detection of Trends in Disturbance and Recovery (LandTrendR) (KENNEDY; YANG; COHEN, 2010) e o Vegetation Regeneration and Disturbance Estimates through Time (VeRDET) (HUGHES; KAYLOR; HAYES, 2017) podem ser particularmente interessantes para análises de regeneração florestal na Amazônia, uma vez que ambos usam como dados de entrada variáveis obtidas a partir de composições anuais de imagens de satélite. Apesar de não resultarem diretamente em trajetórias de uso e cobertura da terra, esses algoritmos também classificam os segmentos temporais em "distúrbio", "estável" ou "regeneração", com base na variação dos valores analisados.

Para efeito de classificação de trechos de não mudança, podem-se utilizar classificadores supervisionados capazes de lidar com dados de alta dimensionalidade, como o Random Forest e o Support Vector Machine (SVM), aqueles baseados em redes neurais, ou ainda algoritmos baseados em distâncias de séries temporais, dos quais o Dynamic Time Warping (DTW) é um dos mais conhecidos. Segundo Maus et al. (2016), o DTW distorce o tempo para ajustar as duas séries a serem comparadas, podendo não ser apropriado para análises de sensoriamento remoto, em que o tempo decorrido entre as observações é um fator importante. Uma solução para esse problema é a extensão do DTW ponderada pelo tempo, o TimeWeighted Dynamic Time Warping (TWDTW) (MAUS et al., 2016). O TWDTW demonstra grande potencial 
para a discriminação entre classes de pastagem, agricultura, e florestas, por exemplo, em função da variação característica da resposta espectral dos alvos ao longo do ano. No entanto, como séries temporais de classes de floresta primária e vegetação secundária podem ser muito semelhantes, a distinção entre essas classes por esse método pode não ser adequada. O TWDTW está atualmente disponível no pacote SITS (Satellite Image Time Series Analysis), desenvolvido na linguagem R (E-SENSING, 2019). Além do TWDTW, o SITS reúne ferramentas próprias para a classificação de trajetórias de uso e cobertura da terra a partir de séries temporais de imagens de sensoriamento remoto com grande número de observações. Constam nesse pacote ferramentas que possibilitam a importação de séries temporais de sensoriamento remoto diretamente de web services; a visualização, o agrupamento e a filtragem dessas séries; e métodos de classificação de séries temporais de sensoriamento remoto baseados em aprendizado de máquina. Para casos em que se dispõe de apenas uma imagem por data de interesse (séries temporais esparsas), os classificadores contidos no SITS tendem a se comportar como classificadores supervisionados tradicionais. Atualmente, o SITS não está equipado para lidar com transições inválidas.

Usualmente, transições inválidas em conjuntos multitemporais de classificações de cobertura da terra são tratadas como erros de classificação e mascaradas da análise, ou corrigidas em etapas de pósclassificação. Formas de corrigir essas transições incluem edição manual e utilização de filtros temporais (GRIFFITHS; JAKIMOW; HOSTERT, 2018; SOUZA et al., 2020). Em particular, uma forma relativamente simples de impedir a ocorrência de transições inválidas no processo de classificação é o uso de máscaras, como é o caso da metodologia adotada pelo Programa de Monitoramento do Desmatamento da Floresta Amazônica Brasileira por Satélite (PRODES). Como áreas previamente desmatadas ou que originalmente não eram cobertas por florestas são mascaradas da análise no PRODES, apenas desmatamentos de florestas primárias são contabilizados (CÂMARA; VALERIANO; SOARES, 2006) e transições inválidas, como uma área desflorestada se regenerar para uma floresta primária, não são mapeadas.

Outra forma comumente utilizada para classificar trajetórias de uso e cobertura é gerar uma classificação de determinada data, como classificação base, e atualizá-la para a próxima data utilizando técnicas de detecção de mudanças bitemporais binárias (COMBER; LAW; LISHMAN, 2004; XIAN; HOMER, 2010; HUANG et al., 2017). Por envolver diversas etapas e processos, esse método pode demandar muito esforço e tempo por parte do analista, principalmente para análises que envolvem grande número de observações. Erros de classificação também se propagam na análise.

Também é possível coletar amostras das trajetórias de interesse, geralmente em um trecho da série temporal de imagens, e usar essas amostras para definir as regras de identificação das trajetórias esperadas ou treinar classificadores para detecção dessas trajetórias em toda a série temporal (ZHU, 2017; ANJOS et al., 2015). Segundo Zhu (2017), esse método possui alto custo computacional e necessita de conhecimento prévio sobre os tipos de mudanças a serem detectados.

Recentemente, observou-se a proposição de algoritmos baseados em processos de Markov para a classificação de trajetórias de uso e cobertura da terra. Esses algoritmos incorporam tanto os valores do pixel sendo classificado como informações acerca da probabilidade de ocorrência conjunta entre classes ordenadas no tempo. Como exemplos desse tipo de proposta, são citados o Virtual Land Cover Engine (VLCE) (HERMOSILLA et al., 2018) e o Compound Maximum a Posteriori (CMAP) (REIS et al., 2020). O VLCE é um conjunto de algoritmos utilizados para incorporar a dimensão temporal em uma extensão do conhecido modelo de contexto espacial Iterated Conditional Modes (ICM). Essa extensão permite a incorporação do conhecimento sobre as probabilidades de transições para corrigir classificações de cobertura obtidas por Random Forest. Trata-se de um algoritmo iterativo, em que são necessários vários passos de classificação, incluindo o cálculo de probabilidades de sucessões de classes distintas antes e depois do evento de mudanças mapeado externamente ao VLCE.

O CMAP é um classificador do tipo generativo que incorpora os valores dos pixels observados em todos os pontos da série temporal e a distribuição conjunta das classes no tempo. No CMAP, as trajetórias de uso e cobertura são consideradas como uma cadeia de Markov, de forma que as probabilidades entre transições podem ser globalmente modeladas por uma matriz de probabilidades de transição. Essa matriz é estimada a partir de dados auxiliares, ou por valores definidos pelo analista. O CMAP classifica a série 
temporal de imagens de sensoriamento remoto diretamente em trajetórias válidas de uso e cobertura em passo único, e em uma localidade por vez. Essas características permitem algumas vantagens específicas para estudos de regeneração florestal, como a possibilidade de incluir a informação multitemporal dos dados para a distinção entre áreas de florestas primárias e de vegetação secundária, mesmo que o evento de desmatamento não esteja registrado nos dados utilizados (REIS et al., 2020). Como a classificação de todas as datas é feita de forma simultânea, erros de classificação não se propagam nas trajetórias, como pode ocorrer em métodos que dependem de sistemas de regras para a eliminação de trajetórias inválidas. Também é possível utilizar dados multissensores e diferentes níveis de legenda para cada data e/ou tipo de dado, característica importante para análises de áreas e/ou períodos com baixa disponibilidade de dados de um mesmo sensor.

\subsubsection{ANÁLISE DA QUALIDADE}

Esta etapa ainda apresenta grandes desafios de implementação, uma vez que geralmente depende da existência de dados de referência, usualmente obtidos a partir de informações coletadas em campo, imagens de melhor resolução espacial e/ou dados auxiliares. Esse tipo de dado pode ser difícil de adquirir para estudos multitemporais envolvendo várias observações, uma vez que depende da coleta sistemática de dados para mesma área de estudo ao longo do tempo (FOODY, 2010; LU; LI; MORAN, 2014). De forma geral, as análises de qualidade das trajetórias se baseiam no cálculo de índices de exatidão derivados de matrizes de contingência, também conhecidas como matrizes de confusão ou de erro. É comum que as classificações de uso e cobertura de cada data sejam avaliadas de forma independente, como proxy da avaliação da exatidão da trajetória. Nesses casos, em função da falta de dados de referência para algumas datas, geralmente as mais antigas, por vezes se admite que a exatidão de uma classificação seja adotada como a exatidão das demais classificações obtidas com mesmas classes, dados e métodos, mas em datas distintas, o que pode não ser verdade. Segundo Sexton et al. (2013), erros de classificação não são constantes ao longo dos anos, mesmo com uso das mesmas técnicas e dados, em função de variações intrínsecas de cada data, seja na cobertura da terra ou nas imagens. Para efeito de cálculo de índices de exatidão para todas as datas, alguns estudos se concentram na coleta de amostras de referência a partir das próprias imagens de sensoriamento remoto utilizadas para classificação. Nesses casos, deve-se atentar que os índices de exatidão calculados provavelmente estarão superestimados. Existem diferentes ferramentas que podem facilitar o processo de seleção de amostras de referência, incluindo aquelas especialmente desenvolvidas para observação de séries temporais. Algumas dessas ferramentas estão analisadas em Jakimow et al. (2020).

Independente da ferramenta selecionada, um passo crucial para a análise da qualidade das trajetórias é definir qual aspecto das trajetórias está sendo avaliado. Para alguns casos, apenas a classificação correta de determinada sequência de transições é o suficiente, não importando o momento exato da observação de classes/mudanças específicas. Outras análises podem demandar a correta observação de cada classe em cada tempo. Em particular, deve-se também atentar para problemas de classificação em áreas de transição de classes. Mesmo para a análise de uma classificação de uso e cobertura da terra em uma única data, é possível que as classes utilizadas representem estágios em um gradiente, com limites pouco precisos entre essas classes (POWELL et al., 2004). Powell et al. (2004), por exemplo, analisaram fontes de erros em classificações de uso e cobertura da terra baseadas em imagens Landsat 5/TM, em uma área em Rondônia. Segundo os autores, as classes dominantes da área de estudo se apresentavam em um gradiente entre pasto e vegetação secundária. Amostras de referência foram obtidas a partir de imagens de alta resolução, por meio da análise visual de cinco intérpretes treinados. Esses intérpretes discordaram da classificação em quase 30\% das amostras, principalmente naquelas localizadas em áreas de borda entre feições, nas de pixel misto (pixels recobrindo mais de uma classe) ou em áreas em transição (classes sobrepostas no gradiente), além de problemas de correção geométrica (POWELL et al., 2004). Assim, a própria confiabilidade das amostras de referência deve ser avaliada e considerada na análise, como demonstrado por Foody (2010) e Olofsson et al. (2014).

Para análises envolvendo muitas datas, no entanto, espera-se que as classificações anteriores e posteriores à(s) data(s) de transição não sejam afetadas por esse tipo de problema. Se o momento exato em 
que uma área passou de uma classe para a classe seguinte do gradiente não for importante, a trajetória obtida é similar, independente de qual das duas classes é atribuída para a área em transição. Dessa forma, erros de classificação em cada data não necessariamente implicam em erros nas trajetórias. Como a regeneração florestal é um processo contínuo, é natural que as classes envolvidas na análise representem um gradiente, sendo que áreas de agricultura em pousio/de pasto sujo podem ser consideradas como classes de transição entre agricultura/pasto e vegetação secundária. Por outro lado, classes de vegetação secundária também podem ser divididas em diferentes estágios de desenvolvimento, e a classificação incorreta de um estágio em determinada data pode não afetar a análise da trajetória em si. Na análise de trajetórias de regeneração florestal, deve-se atentar para possíveis problemas de definição de classes (quando uma área efetivamente se torna vegetação secundária e em qual estágio de desenvolvimento) e de classificação (capacidade de discriminação entre essas classes). Essa característica prejudica o estabelecimento da data exata de formação da vegetação, mesmo em estudos com mapeamentos em todas as datas de interesse. Assim, maior atenção deve ser dada para análises que objetivam quantificar áreas de vegetação secundária em cada data ou que objetivam calcular a idade da vegetação secundária.

Outras possibilidades de avaliação de qualidade das trajetórias encontradas na literatura analisam trajetórias tendo como base regras lógicas da probabilidade de transição de uma classe para a outra, sem a utilização de amostras de referência (LIU; ZHOU, 2004; AZEREDO et al., 2016). Este tipo de análise é particularmente útil para identificar erros provenientes de transições inválidas, que podem ser representadas em um mapa de validade das transições (REIS et al., 2020).

Apesar de não se tratar de um indicativo direto de exatidão, também é possível avaliar a qualidade das classificações por meio de análises de incerteza (REIS et al., 2017). Um exemplo desse tipo de análise consiste em variar a abordagem de classificação de cobertura (classificador, parâmetros, conjunto de amostras de treinamento) e contabilizar variações nos resultados de classificação de cobertura/trajetórias (i.e. identificação de pixels de classificação instável). Considerando classificadores supervisionados, é comum que os algoritmos atribuam as classes para cada pixel/objeto em função do menor ou maior valor de alguma medida calculada para cada classe, como probabilidade ou distância. Para resultados obtidos utilizando esses classificadores, Wulder et al. (2018) mencionam também a possibilidade de gerar mapas de qualidade com a diferença entre os valores dessas medidas para as classes ordenadas em primeira e segunda posição. Quanto menor essa diferença, maior seria a incerteza envolvida na classificação. Outros exemplos de índices de incerteza para esse tipo de análise estão apresentados e discutidos em Gonçalves et al. (2009).

\subsection{Extração e análise de informações}

As trajetórias de uso e cobertura da terra podem ser utilizadas, por exemplo, para identificar a data em que ocorreu uma mudança (ZHU et al., 2016), descrever a porcentagem ocupada por cada classe de cobertura em cada data analisada, área alterada e tipos de transições observadas (YUAN et al., 2005; SEXTON et al., 2013; HERMOSILLA et al., 2015; ZOUNGRANA et al., 2015; FRANKLIN et al., 2015; HERMOSILLA et al., 2018) e para analisar a ocorrência e os padrões espaciais de cada tipo de trajetória (MENA, 2008; MÜLLER-HANSEN et al., 2017). Dentre os métodos usualmente utilizados, citam-se a interpretação visual dos resultados e o cálculo de índices próprios para caracterização de feições em uma ou duas datas consecutivas. Como exemplo de índices, são citados índices de fragmentação, porcentagem/área ocupada por cada classe de cobertura da terra e/ou de mudança, número de feições de uma dada cobertura/mudança, forma das feições, distância entre feições, razão área-perímetro, índice de diversidade de Shannon, entre outros (GILLANDERS et al., 2008). Ressalta-se, no entanto, que a comparação entre informações individuais de cada data ou de mudanças bitemporais não configuram análises de trajetórias em si. Apesar do potencial de gerar resultados importantes, grande parte da informação temporal contida nas trajetórias é ignorada devido a esse tipo de simplificação.

Como exemplo de aplicação de análises para cada data e pares de datas em estudos de regeneração florestal, Carvalho et al. (2019) avaliaram mudanças no padrão histórico de acumulação da vegetação secundária no estado do Pará para anos entre 2004 e 2014, utilizando dados do projeto Mapeamento do Uso e Cobertura da Terra nas Áreas Desflorestadas da Amazônia Legal (TerraClass). Detalhes sobre esses dados 
estão apresentados na Seção 3. Segundo os autores, houve um aumento na conversão de vegetação secundária para pastos limpos e agricultura mecanizada, principalmente após 2010, que se trata de um período em que se observa redução de taxas de desmatamento de florestas primárias. No mesmo período, os autores identificaram uma diminuição de áreas de classes com suposto maior potencial de regeneração: mosaicos de ocupação e pastos em regeneração. Assim, os autores discutem que é possível que se estabeleça uma tendência de diminuição de áreas de vegetação secundária nos anos seguintes ao da análise. Adicionalmente, os autores encontraram diferenças na concentração espacial de vegetação secundária nas regiões norte e sul do estado, cujos históricos de ocupação e taxas de desmatamento de florestas primárias diferem.

Das trajetórias, é possível extrair informações sobre a idade da vegetação secundária (NUNES et al., 2020; SILVA JUNIOR et al., 2020) e também da duração e/ou intensidade dos processos, que são de particular interesse para análises de regeneração florestal. Por exemplo, Müller et al. (2016) e Jakovac et al. (2017) extraíram o número e a duração de ciclos de regeneração florestal a partir de séries temporais de dados Landsat com mais de 29 anos de dados. Müller et al. (2016) observaram diferenças na duração e frequência dos ciclos de regeneração ao longo da BR-163, entre os estados do Pará e do Mato Grosso, dependendo do tipo de uso predominante e da proximidade de florestas. Esse estudo foi realizado com base na detecção de áreas desmatadas e posterior mapeamento de áreas de vegetação secundária. Jakovac et al. (2017), com uso do algoritmo Breaks For Additive Season and Trend(BFAST) (VERBESSELT et al., 2010) e Random Forest, segmentaram e classificaram uma série temporal de imagens dos municípios de Tefé e Alvarães, no estado do Amazonas. Os autores observaram uma diminuição média no tempo dos ciclos de regeneração em áreas de agricultura itinerante entre os períodos de 1987-2000 e 20012014, o que indica a existência de um processo de intensificação agrícola na região (JAKOVAC et al., 2017).

Não obstante, um mesmo estudo pode reunir análises em cada data, em pares de datas e de trajetórias. Como exemplo desse tipo de estudo, Wang et al. (2020) utilizam dados TerraClass para toda a Amazônia Legal Brasileira no período de 2000 a 2014 para analisar o desmatamento de vegetação secundária, pela comparação de pares de dados consecutivos. Essa análise se deu de forma estratificada em função de categorias de idade da vegetação secundária, que foram determinadas a partir do empilhamento dos dados TerraClass. Os autores observaram duas fases distintas de desmatamento de vegetação secundária: 1) entre 2000 e 2008 e 2) entre 2008 e 2014. No primeiro período, há uma diminuição na taxa de desmatamento de vegetação secundária que acompanha a diminuição do desmatamento de florestas primárias. O segundo período é caracterizado pelo aumento do desmatamento de vegetação secundária paralelo à diminuição do desmatamento de florestas primárias. Os autores sugerem que no segundo período houve aumento da pressão sobre sistemas florestais, que foi absorvida por áreas em regeneração. Os autores também concluem que $91 \%$ das áreas de vegetação secundária são convertidas para pasto, independente da idade dessa vegetação.

Outra pergunta comum em estudos de trajetórias se refere aos principais tipos de trajetórias existentes, que podem se diferenciar pela duração/intensidade dos processos ou pela composição das trajetórias, ou seja, pela sequência temporal de classes de uso e cobertura da terra. Foram identificados dois métodos principais para a definição de tipos de trajetórias baseados na composição dessas trajetórias. O primeiro deles é a definição exaustiva de tipologias baseadas nos processos observados (PINHEIRO et al., 2016; CORSINI, 2018; ASSIS et al., 2020). O segundo método é a utilização de algoritmos automáticos de agrupamento, que podem ajudar a identificar grupos de pixels com mesmo padrão espaço-temporal (AZEREDO, 2017).

Como exemplo do primeiro método, Corsini (2018) observou diferenças no padrão de biomassa acima do solo na Amazônia Legal Brasileira, e dos impactos da ocorrência de fogo e déficit hídrico nesses padrões, dependendo do tipo de trajetória de regeneração florestal. Essas trajetórias foram definidas em função da observação de determinadas sequências de classes em mapeamentos do projeto TerraClass para os anos de 2004, 2008 e 2010. Esse tipo de análise é viável para poucas datas. Com o aumento das datas analisadas, espera-se também um aumento no número de trajetórias possíveis, que podem envolver diferentes ciclos de abandono, regeneração e desmatamento. Como trajetórias de regeneração florestal são bastante diversas e variam no tempo e no espaço, identificar todas as possíveis tipologias a priori é uma tarefa com alto grau de complexidade, o que pode ser custoso para o analista e resultar em análises subjetivas e/ou sintéticas, com foco em apenas alguns processos previamente identificados. 
Em relação ao segundo método, Azeredo (2017) propôs um algoritmo próprio para a análise da evolução temporal de trajetórias de uso e cobertura da terra, denominado Grouping by Similarity of Temporal Evolution (GSTE), a partir de uma metodologia que combina os algoritmos DTW, Classical Multidimensional Scaling (CMDS) e K-Means Clustering. Segundo o autor, os agrupamentos resultantes retratam características como o intervalo de tempo entre diferentes processos, a duração de cada processo e classes envolvidas em cada tempo. Ressalta-se que, enquanto trajetórias de uso e cobertura da terra são geralmente apresentadas em categorias, a versão atual do GSTE é aplicável apenas para dados numéricos. Assim, classes de uso e cobertura precisam ser convertidas para valores associados a diferentes intensidades de um único processo para análise, $o$ que pode não ser diretamente derivável para análises que envolvem múltiplos processos. Diferentes métodos categóricos de agrupamento têm sido propostos na literatura, como o K-Modes (HUANG, 1998), o RObust Clustering using linKs (ROCK) (GUHA; RASTOGI; SHIM, 2000) e o Generalized Self-Organizing Maps (GSOM) (HSU, 2006). Até o momento, não foram encontrados exemplos de aplicação de algoritmos automáticos de agrupamento para análise de trajetórias de regeneração florestal.

Alguns exemplos de trajetórias de uso e cobertura da terra estão ilustrados na Figura 2. Nessa figura, três trajetórias caracterizam ciclos de regeneração florestal $\left(S_{1}, S_{2}\right.$ e $\left.S_{3}\right)$. A partir dessas trajetórias, é possível calcular o valor de três atributos: tempo de uso antes da regeneração, persistência da vegetação secundária, e duração do ciclo de regeneração. Estão também ilustradas trajetórias que não caracterizam um ciclo de regeneração florestal $\left(\mathrm{S}_{4}\right)$ e trajetórias inválidas $\left(\mathrm{S}_{5}\right)$. Algumas das trajetórias apresentam a mesma sequência de eventos com durações distintas, apresentando também diferentes valores para cada atributo $\left(S_{1}\right.$ e $\left.S_{2}\right)$. Outras trajetórias apresentam sequências de eventos diferentes, o que pode impactar na adoção de tipologia e na interpretação dos resultados, mesmo apresentando valores idênticos em seus atributos $\left(S_{2}\right.$ e $\left.S_{3}\right)$. Para esses exemplos hipotéticos, variações nos métodos de análise podem resultar em interpretações muito distintas dos processos de mudança de uso e cobertura da terra em uma dada área de estudo.

Figura 2 - Exemplos de trajetórias de uso e cobertura da terra.

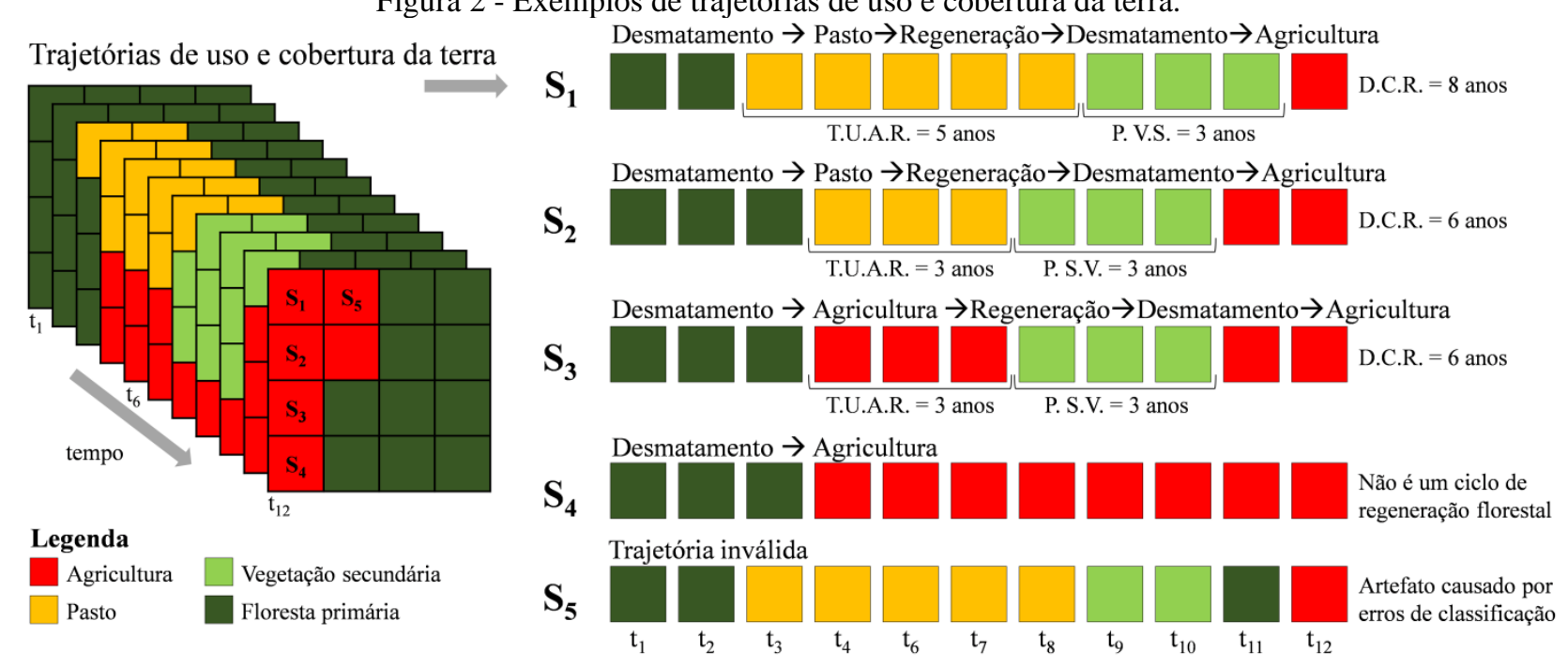

Em que: $t_{i}=$ ano da observação; T.U.A.R. = Tempo de Uso Antes da Regeneração; P.V.S.= Persistência da Vegetação Secundária; D.C.D. = Duração do Ciclo de Regeneração Florestal. Um ciclo de regeneração florestal começa com um evento de desmatamento (de floresta primária ou vegetação secundária) e termina com o desmatamento da vegetação secundária. Esses atributos foram definidos pelos autores para exemplificação.

Fonte: Os autores (2020).

Técnicas próprias para análises de trajetórias de uso e cobertura da terra foram apresentadas por Azeredo et al. (2016) e Maciel et al. (2019). Azeredo et al. (2016) adaptaram os padrões clássicos encontrados na literatura de objetos móveis para o contexto de análise de trajetórias de uso e cobertura da terra, com estudos de caso focados em análises de degradação florestal. Apesar de ainda não terem sido aplicadas para análises de regeneração florestal, essas ferramentas podem ser utilizadas, por exemplo, para identificar: 
a) trajetórias que convirjam para classes específicas, sendo que essas podem denotar um processo de regeneração florestal;

b) trajetórias que possuam determinadas classes de uso e cobertura em janelas temporais específicas. Essa ferramenta permite a identificação de vegetação secundária em determinados períodos e não em datas individuais, amenizando problemas de classificação em áreas em transição;

c) trajetórias que apresentem mudanças de cobertura similares, no mesmo período ou não. Utilizável para a identificação desmatamento de áreas de vegetação secundária para certos tipos de uso;

d) trajetórias que apresentem similaridade na intensidade dos processos, como por exemplo aquelas que apresentam similaridade no tempo de pousio/regeneração;

e) trajetórias que apresentem transições inconsistentes/inválidas.

Maciel et al. (2019) formalizaram uma lógica de cálculo espaço-temporal denominada LUC Calculus. Essa ferramenta permite que o analista investigue e caracterize trajetórias de uso e cobertura da terra em função de quatro predicados principais: conversão (CONVERT), recorrência (RECUR), evolução (EVOLVE) e manutenção $(H O L D)$. Estes predicados, adaptados ao contexto da análise de regeneração florestal, são interpretados por:

a) CONVERT denota a mudança entre classes em dois tempos consecutivos. Exemplo de utilização: detectar todas as áreas de vegetação secundária que foram convertidas para uso agrícola;

b) RECUR denota a observação de classe recorrente após essa classe ter sido convertida para outra classe. Exemplo de utilização: corrigir transições inválidas e distinção de áreas de vegetação secundária e florestas primárias pela análise da trajetória;

c) EVOLVE denota eventos em que há uma ou mais classes diferentes intercaladas entre a mudança de uma classe para outra. Exemplo de utilização: identificar áreas de florestas primárias que foram desmatadas e abandonadas e encontram-se como vegetação secundária, independente do uso intermediário;

d) HOLD denota uma classe que permaneceu a mesma em todas as observações de um intervalo de tempo. Exemplo de utilização: identificar áreas de vegetação secundária persistente.

\section{MAPEAMENTOS DISPONÍVEIS}

Existem diferentes programas de monitoramento de florestas tropicais, em escala local ou global, que geram dados de mudanças de uso e cobertura para a Amazônia e que podem ser incluídos na análise como dados auxiliares. Dentre eles, podem ser citados programas focados em desflorestamento e/ou degradação florestal, como o PRODES (INPE, 2020c), o Sistema de Detecção de Desmatamento em Tempo Real (DETER) e programas incorporados ou derivados, como o Mapeamento da Degradação Florestal na Amazônia Brasileira (DEGRAD) e o DETER intenso (INPE, 2020d), o Sistema de Alerta de Desmatamento (SAD) do Instituto Homem e Meio Ambiente da Amazônia (IMAZON, 2020), e dados do laboratório Global Land Analysis and Discovery (GLAD) como o Global Forest Change dataset (HANSEN et al., 2013). Também é possível utilizar mapeamentos de recursos naturais, como os pioneiros RADAM (Radar na Amazônia) e RADAMBRASIL (VAN ROESSEL; GODOY, 1974), bem como mapeamentos multitemporais de uso e cobertura da terra, como o Monitoramento da Cobertura e Uso da Terra do Brasil (IBGE, 2015,2017,2020). De particular interesse para análises de regeneração florestal, no entanto, são os projetos que mapeiam classes de uso e cobertura em áreas desmatadas, sendo crucial a identificação de áreas de vegetação secundária. Dentre os mapeamentos desse tipo na Amazônia brasileira, são citados o TerraClass e o Projeto de Mapeamento Anual da Cobertura e Uso do Solo no Brasil (MapBiomas). As principais características desses projetos estão resumidas a seguir.

O projeto TerraClass é desenvolvido pelo Instituto Nacional de Pesquisas Espaciais (INPE), em parceria com a Empresa Brasileira de Pesquisa Agropecuária (Embrapa). As áreas desflorestadas mapeadas 
pelo PRODES na Amazônia Legal Brasileira são classificadas em classes de interesse. Atualmente, estão disponíveis mapeamentos para os anos de 2004, 2008, 2010, 2012 e 2014. Wang et al. (2020) mencionam ainda o uso de dados TerraClass de 2000, não disponibilizados via sítios eletrônicos do projeto (http://www.inpe.br/cra/projetos_pesquisas/dados_terraclass.php ou https://www.terraclass.gov.br/). O projeto TerraClass se baseia na classificação de imagens da família Landsat (ou de resolução similar) e dados do Moderate-Resolution Imaging Spectroradiometer (MODIS), utilizando diferentes técnicas de processamento de imagens e etapas de análise visual, edição manual e auditoria. Os dados estão disponibilizados em duas versões de legenda, ambos em $30 \mathrm{~m}$ de resolução espacial e com três mapeamentos de cobertura originários dos dados PRODES (Hidrografia, Não floresta e Floresta). Na primeira versão, são mapeadas pelo TerraClass 13 classes de cobertura: Desflorestamento do ano, Agricultura anual, Pasto com solo exposto, Pasto limpo, Pasto sujo, Regeneração com pasto, Vegetação secundária, Reflorestamento (com exceção do mapeamento de 2008), Área urbana, Mineração, Mosaico de ocupações, Outros e Área não observada (ALMEIDA et al., 2016). Almeida et al. (2016) calcularam uma Exatidão Global de 76,6\% e índice Kappa de 0,67 para os mapeamentos do Pará e Mato Grosso de 2008, com amostras de campo das classes Agricultura anual, Mosaico de ocupações, Pasto limpo, Pasto sujo, Regeneração com pasto e Vegetação secundária. Nesses dados, a maior confusão ocorre entre classes de pasto. Os valores de Exatidão Global e Kappa aumentam respectivamente para 89,7\% e 0,79 ao mesclar as 3 classes de pasto (Pasto limpo, Pasto sujo, Regeneração com pasto). Na segunda versão de legenda, são classificadas: Vegetação natural florestal secundária, Desflorestamento do ano, Cultura agrícola perene, Cultura agrícola semiperene, Cultura agrícola temporária, Pastagem cultivada arbustiva, Pastagem cultivada herbácea, Silvicultura, Área urbanizada, Mineração, Não observado, e Outros.

Como o projeto TerraClass observa apenas as áreas desmatadas detectadas pelo PRODES, de confiabilidade internacionalmente reconhecida, áreas de vegetação secundária são mapeadas apenas em áreas previamente desmatadas (ALMEIDA et al., 2016). Esse aspecto metodológico diminui consideravelmente a confusão entre áreas de florestas e vegetação secundária. No entanto, esses dados não são anuais e abrangem um período relativamente curto, de forma que alguns ciclos de regeneração mais longos não seriam observados pela análise baseada apenas nesse conjunto de dados. Além disso, dados TerraClass possuem problemas de desalinhamento herdados do PRODES (INPE, 2017; WANG et al., 2020), que devem ser corrigidos para análises no nível do pixel. Dessa forma, esses dados são indicados para caracterização de trajetórias curtas, como demonstrado em Corsini (2018) e em Wang et al. (2020) e detalhados na Seção 2.3, ou ainda como dado auxiliar para processos de classificação de trajetórias de uso e cobertura da terra: por exemplo, como máscara de classificação, classificação base a ser atualizada, e/ou para coleta de amostras de referência.

O projeto MapBiomas é produzido por uma rede colaborativa formada por diferentes instituições. Esse projeto objetiva produzir classificações anuais de uso e cobertura da terra para todo o Brasil, utilizando técnicas automáticas para classificar imagens da família Landsat. De forma geral, mosaicos construídos a partir de variáveis estatísticas (mediana, amplitude, desvio padrão, entre outras) de conjuntos de imagens de cada ano são classificados de forma independente. Transições inválidas são então corrigidas no processo de pós-classificação, com uso de filtros espaciais e/ou temporais baseados em regras pré-estabelecidas (SOUZA et al., 2020). As imagens, classes, exatidão do mapeamento e detalhes metodológicos variam dependendo da coleção. Os valores de Exatidão Global são estimados a partir de amostras coletadas por meio de interpretação visual das imagens. Apenas informações gerais sobre a coleta de amostras estão contidas na documentação das coleções (Documento Base Teórico do Algoritmo - ATBD), que também está em constante alteração. Até o momento (novembro de 2020), foram publicadas oito coleções. A partir da coleção 3.x não há distinção entre classes de florestas primárias, degradadas ou de vegetação secundária (MAPBIOMAS, 2020).

A coleção 2.x, última em que havia distinção entre florestas primárias e vegetação secundária, possui dados anuais entre 2000 e 2016, com índices de Exatidão Global para a classificação de cada ano variando em torno de $80 \%$ para a Amazônia. Neves et al. (2020), após uma etapa de harmonização de legendas entre os dados TerraClass (primeira legenda) e da coleção 2 do MapBiomas, compararam os resultados de classificação entre as áreas de sobreposição dos mapeamentos de 2004, 2008, 2010, 2012 e 2014. Considerando todos os anos em conjunto, os autores constataram que apenas 3,56\% das áreas mapeadas como vegetação secundária no TerraClass foram mapeadas como a classe correspondente no MapBiomas. O 
restante da área foi mapeada majoritariamente como Floresta $(80,34 \%)$ e Pasto $(13,96 \%)$. Apesar dessas diferenças, como a classe predominante em ambos os mapeamentos é a de floresta, essas classificações apresentam concordância de classificação em torno de $87 \%$ dos pixels, o que pode se refletir nos valores de Exatidão Global apresentados pelos dados do MapBiomas. Dessa forma, apesar de os dados da coleção 2 do MapBiomas permitirem análises mais longas e anuais, deve-se considerar as possíveis confusões entre áreas de floresta primária e vegetação secundária.

Para uso das coleções mais recentes do MapBiomas para análises de regeneração florestal, é possível reclassificar como vegetação secundária as florestas que ocorrem em áreas previamente desmatadas (NUNES et al. 2020; SILVA JUNIOR et al., 2020). Nesse caso, a identificação do evento de desmatamento depende da observação de uma classe diferente de floresta em uma data anterior. Nunes et al. (2020) usaram essa abordagem para separar áreas de florestas primárias e vegetação secundária nos dados MapBiomas da coleção 3.1, para o bioma Amazônia. A partir desses dados, os autores agruparam as áreas de vegetação secundária por idade estimada e calcularam a área ocupada/ desmatada da vegetação secundária no bioma, bem como realizaram análises de estimativa de captura de carbono. No entanto, essa abordagem é incapaz de distinguir áreas de vegetação secundária caso não haja evidências de desmatamento durante a série histórica. Por esse motivo, Nunes et al. (2020) encontraram proporções de áreas desmatadas ocupadas por vegetação secundária menores que o TerraClass para períodos correspondentes. Utilizando abordagem similar, Silva Junior et al. (2020) criaram um banco de dados contendo valores de incremento, extensão e idade da vegetação secundária para os anos de 1986 a 2018, a partir de dados da coleção 4.1 do MapBiomas. Esses resultados também foram validados utilizando dados TerraClass, por um método que compara a proporção da classe em uma grade celular regular. Os autores concluíram que apesar da proporção média de vegetação secundária encontrada pelo processamento dos dados MapBiomas ser significativamente menor que a dos dados TerraClass (via teste de Mann-Whitney e p-valores menores que 0,001) os resultados dos dois conjuntos de dados são comparáveis. Além desses trabalhos, um módulo de análise de regeneração florestal nativo do projeto MapBiomas se encontra em construção, mas até o momento não há informações disponíveis acerca da exatidão das classes de vegetação secundária.

Segundo Maurano e Escada (2019), as variações nos mapeamentos históricos do MapBiomas para cada coleção limitam a aplicabilidade desses dados para atividades que exigem mapeamentos operacionais contínuos e precisos para estimativas de áreas. Em termos de análises de regeneração florestal, essas variações implicam na obtenção de trajetórias distintas, das quais diferentes taxas de absorção de carbono da atmosfera podem ser calculadas, por exemplo. Problemas na identificação de áreas de vegetação secundária quando não há evidência de eventos de desmatamento também podem ser prejudiciais para análises em regiões dominadas por vegetação secundária mais antiga, como no nordeste do estado do Pará (CAPANEMA et al., 2019). Assim, dados MapBiomas são adequados para análises preliminares e em áreas extensas, a serem refinadas para estudos de regeneração florestal mais detalhados.

\section{CONSIDERAÇÕES FINAIS}

Os principais dados e métodos para mapeamento e análise de trajetórias de uso e cobertura da terra foram revisados no presente artigo e discutidos em função da aplicabilidade para estudos de regeneração florestal na Amazônia brasileira. De forma geral, observou-se que as análises podem ser baseadas: 1) no uso sucessivo de métodos próprios para pares de imagens ou 2) na utilização de técnicas e ferramentas próprias para análises de séries temporais de imagens de sensoriamento remoto e trajetórias de uso e cobertura da terra.

Os estudos e produtos de sensoriamento remoto revisados nesse trabalho baseiam-se principalmente em dados da família Landsat. Esses dados estão disponíveis para uso com correções atmosféricas e geométricas. Apesar dos avanços na disponibilidade de imagens e em técnicas de composição de imagens, a cobertura de nuvens ainda representa desafios científicos importantes para o Bioma Amazônia. O desenvolvimento de métodos que combinam imagens de radar com imagens ópticas são de particular importância para estudos de trajetórias nessa região, constituindo-se em uma alternativa possível para aumentar o número de observações das séries temporais. Assim, métodos capazes de lidar com séries temporais esparsas, dados multissensores e diferentes níveis de legenda são de particular interesse para 
análises de regeneração florestal. Outro problema recorrente entre as técnicas de classificação de trajetórias é a necessidade de vários passos, além de uma etapa de pós-classificação para correção de trajetórias inválidas. O classificador CMAP se destaca por classificar uma série temporal de imagens de sensoriamento remoto diretamente em trajetórias de uso e cobertura da terra, em um único passo, sendo possível utilizar dados multissensores em variados níveis de legenda. Atualmente o CMAP apresenta alto custo computacional para áreas extensas e/ou séries-temporais longas, o que pode limitar sua utilização de forma operacional. Para estudos futuros, são esperadas melhorias de desempenho do CMAP, bem como a incorporação de novos classificadores base ao algoritmo e métodos de cálculo da probabilidade de transições.

Apesar da diversidade de técnicas e ferramentas propostas para análise de trajetórias de uso e cobertura da terra, os métodos de verificação da qualidade das classificações ainda são majoritariamente baseados no cálculo de índices de exatidão para cada tempo analisado, o que pode não ser o mais indicado para análises de trajetórias. De forma similar, grande parte dos estudos ainda se baseia em análises mono ou bitemporais. Nesse tipo de análise, deve-se atentar para a possível superestimativa dos valores de exatidão, o que pode ser particularmente importante para quantificar áreas de mudanças e/ou de cobertura para cada data. Nesse sentido, um dos grandes desafios na análise de trajetórias de uso e cobertura da terra reside no desenvolvimento de metodologias de cálculo de exatidão e de análise das trajetórias.

Os estudos de regeneração florestal que analisam a informação das trajetórias se resumem naqueles que 1) avaliam poucas datas e/ou classes, e consequentemente poucos tipos de trajetórias, ou 2) que calculam a idade da vegetação secundária. Dessa forma, são necessários estudos voltados para a avaliação de ferramentas para análises de regeneração florestal, principalmente no que se refere a análises com maior número de datas e classes de interesse, bem como aqueles que analisam de forma mais compreensiva trajetórias mais longas e com maior número de classes, para diferenciação de processos até então não discriminados. Em particular, é importante quantificar características próprias dos processos de regeneração florestal, como persistência/idade da vegetação secundária e intensidade, tipo, e tempo de uso antes do abandono em áreas com diferentes históricos de ocupação e sob a influência de diferentes políticas públicas. Esse tipo de estudo tem o potencial de melhorar a precisão e exatidão das estimativas de emissão e balanço de carbono. Também são importantes para estudos de biodiversidade, uma vez que áreas de vegetação secundária podem conectar manchas florestais. Do ponto de vista socioeconômico, esses estudos podem revelar como as diferentes formas de ocupação produzem diferentes paisagens e dinâmicas de regeneração florestal, o que se trata de importante subsidio para elaboração políticas de conservação, ordenamento territorial e de incentivo às práticas sustentáveis de uso da terra.

\section{Agradecimentos}

Este trabalho foi financiado em parte pela Coordenação de Aperfeiçoamento de Pessoal de Nível Superior (CAPES) e em parte pelo Conselho Nacional de Desenvolvimento Científico e Tecnológico (CNPq), bolsa \#309135/2015-0. Os autores gostariam de agradecer a Noeli Aline Particcelli Moreira, pela ajuda pontual com o resumo de informações, a Eliana Pantaleão por sugestões no texto, e também o Instituto Chico Mendes de Conservação da Biodiversidade (ICMBio) pelas autorizações \#48186-4, \#48186-2 e \#38157-2, o Experimento de Larga Escala na Biosfera-Atmosfera na Amazônia (LBA), e o Projeto de Monitoramento Ambiental por Satélite no Bioma Amazônia, processo \#1022114003005-MSA-BNDES.

\section{Contribuição dos Autores}

Conceptualização: M.S.R, M.I.S.E., S.J.S.S. e L.V.D. Curadoria dos dados: M.S.R. Análise formal: M.S.R.. Aquisição de financiamento: M.S.R, M.I.S.E., S.J.S.S. e L.V.D. Investigação: M.S.R. Metodologia: M.S.R, M.I.S.E., S.J.S.S. e L.V.D. Administração do projeto: M.I.S.E. e S.J.S.S. Recursos: M.I.S.E. e S.J.S.S. Software: M.S.R. Supervisão: M.I.S.E. Validação: M.S.R, M.I.S.E., S.J.S.S.e L.V.D. Visualização: M.S.R. Redação - minuta inicial: M.S.R. Redação - revisão e edição: M.S.R, M.I.S.E., S.J.S.S. e L.V.D. 


\section{Conflitos de Interesse}

Os autores declaram que não há conflitos de interesse.

\section{Referências}

AFFONSO, A. G.; ESCADA, M. I. S.; AMARAL, S.; SOUZA, A. R.; SIQUEIRA, J. M.; TORRES, N. C.; CAMILOTTI, V. L.; DAL'ASTA, A. P.; COSTA, L. C. O.; SOARES, F. R. As comunidades ribeirinhas do Baixo Tapajós (PA): infraestrutura, mobilidade, serviços sócio ambientais e conectividade. São José dos Campos: [s.n.], 2016. Disponível em: <http://urlib.net/sid.inpe.br/mtc-m21b/2016/08.02.12.48>. Acesso em: 21 jul. 2019.

AGUiAR, A. P. D.; OMETTO, J. P.; NOBRE, C.; LAPOLA, D. M.； ALMEIDA, C.; VIEIRA， I. C.; SOARES, J. V.; ALVALA, R.; SAATCHI, S.; VALERIANO, D.; CASTILLA-RUBIO, J. C. Modeling the spatial and temporal heterogeneity of deforestation-driven carbon emissions: the INPE-EM framework applied to the Brazilian Amazon. Global Change Biology, v. 18, n. 11, p. 3346-3366, 2012. DOI. 10.1111/j.1365-2486.2012.02782.x.

ALASKA SATEllite FACILITY (ASF). ALOS PALSAR. 2020. Disponível em: <https://asf.alaska.edu/data-sets/sar-data-sets/alos-palsar/>. Acesso em: 18 dez. 2020.

ALMEIDA, C. A.; $\quad$ COUTINHO, A. C.; $\quad$ ESQUERDO, J. C. D. M.; $\quad$ ADAMI, M.; $\quad$ VENTURIERI, A.; DINIZ, C. G.; DESSAY, N.; DURIEUX, L.; GOMES, A. R. High spatial resolution land use and land cover mapping of the Brazilian Legal Amazon in 2008 using Landsat-5/TM and MODIS data. Acta Amazonica, v. 46, n. 3, p. 291-302, 2016. DOI. 10.1590/1809-4392201505504.

ALMEIDA, C. A.; VALERIANO, D. M.; ESCADA, M. I. S.; RENNÓ, C. D. Estimativa de área de vegetação secundária na Amazônia Legal Brasileira. Acta Amazonica, v. 40, n. 2, p. 289-301, 2010.

ANDERSON, J. R.; HARDY, E. E.; ROACH, J. T.; WITMER, R. E. A land use and land cover classification system for use with remote sensor data. Geologycal Survey Professional Paper 964. United States Government Printing Office: Washington, Estados Unidos da América, 1976. DOI. 10.3133/pp964.

ANJOS, D.; LU, D.; DUTRA, L.; SANT'ANNA, S. Change detection techniques using multisensor data. In: THENKABAIL, P. S. (Ed.) Remotely Sensed Data Characterization, Classification, and Accuracies. London: CRC Press, 2015. v. 1, p. 375-395.

ASSIS, T. O.; AGUIAR, A. P. D.; VON RANDOW, C.; GOMES, D. M. P.; KURY, J. N.; OMETTO, J.; NOBRE, C. A. CO2 emissions from forest degradation in Brazilian Amazonia. Environmental Research Letters, v. 15, n. 10, artigo 104035, 2020. DOI. 10.1088/1748-9326/ab9cfc.

AZEREDO, M. Mineração e análise de trajetórias de mudança de cobertura da terra: explorando padrões comportamentais no contexto da degradação florestal. $150 \mathrm{f}$. Tese (Doutorado em Computação Aplicada) - Instituto Nacional de Pesquisas Espaciais (INPE), São José dos Campos, 2017. Disponível em: <http://urlib.net/sid.inpe.br/mtc-m21b/2017/03.14.13.46>. Acesso em: 24 maio 2018.

AZEREDO, M.; MONTEIRO, A. M. V.; ESCADA, M. I. S.; FERREIRA, K. R.; VINHAS, L.; PINHEIRO, T. F. Mineração de trajetórias de mudança de cobertura da terra em estudos de degradação florestal. Revista Brasileira de Cartografia, v. 68, n. 4, p. 717-731, 2016.

BANSKOTA, A.; KAYASTHA, N.; FALKOWSKI, M. J.; WULDER, M. A.; FROESE, R. E.; WHITE, J. C. Forest monitoring using Landsat time series data: a review. Canadian Journal of Remote Sensing, v. 40, n. 5, p. 362-384, 2014. DOI. 10.1080/07038992.2014.987376.

BIG EARTH OBSERVATION DATA ANALYTICS FOR LAND USE AND LAND COVER CHANGE INFORMATION (E-SENSING). SITS - Satellite Image Time Series Analysis. 2019. Disponível em: <https://github.com/e-sensing/sits/blob/master/README.md>. Acesso em: 05 maio 2019. 
BRASIL. Lei $\mathrm{n}^{\circ}$ 5173, de 27 de outubro de 1966. Dispõe sobre o Plano de Valorização Econômica da Amazônia; extingue a Superintendência do Plano de Valorização Econômica da Amazônia (SPVEA), cria a Superintendência do Desenvolvimento da Amazônia (SUDAM), e dá outras providências. Diário Oficial da União, Brasília, DF, 31 out. 1966. Seção 1, p. 12563.

BRASIL. Lei complementar $n^{\circ}$ 124, de 3 de janeiro de 2007. Institui, na forma do art. 43 da Constituição Federal, a Superintendência do Desenvolvimento da Amazônia - SUDAM; estabelece sua composição, natureza jurídica, objetivos, área de competência e instrumentos de ação; dispõe sobre o Fundo de Desenvolvimento da Amazônia - FDA; altera a Medida Provisória no 2.157-5, de 24 de agosto de 2001; revoga a Lei Complementar no 67, de 13 de junho de 1991; e dá outras providências. Diário Oficial da União, Brasília, DF, 04 jan. 2007. Seção 1, p. 1.

BROWN, S.; LUGO, A. E. Tropical secondary forests. Journal of Tropical Ecology, v. 6, n. 1, p. 1-32, 1990.

CÂMARA, G.; VALERIANO, D. M.; SOARES, J. V. Metodologia para o cálculo da taxa anual de desmatamento na Amazônia Legal. São José dos Campos: INPE, 2006. 24p.

CAPANEMA, V. P.; SANCHES, I. D. A.; ESCADA, M. I. S. Comparação entre os produtos temáticos de uso e cobertura da terra do TerraClass Amazônia e MapBiomas: teste de aderência entre classes. In: SIMPÓSIO BRASILEIRO DE SENSORIAMENTO REMOTO, 19. (SBSR), 2019, Santos. Anais... São José dos Campos: INPE, 2019. p. 724-727. Disponível em: <https://proceedings.science/sbsr2019/papers/comparacao-entre-os-produtos-tematicos-de-uso-e-cobertura-da-terra-do-terraclass-amazoniae-mapbiomas--teste-de-aderenci\#>. Acesso em: 06 out. 2020.

CARVAlHO, R.; ADAMI, M.; AMARAL, S.; BEZERRA, F. G.; AGUIAR, A. P. D. de. Changes in secondary vegetation dynamics in a context of decreasing deforestation rates in Pará, Brazilian Amazon. Applied Geography, v. 106, p. 40-49, 2019.DOI. 10.1016/j.apgeog.2019.03.001

CHAZDON, R. L.; LINDENMAYER, D.; GUARIGUATA, M. R.; CROUZEILLES, R.; BENAYAS, J. M. R.; CHAVERO, E. L. Fostering natural forest regeneration on former agricultural land through economic and policy interventions. Environmental Research Letters, v. 15, n. 4, artigo 043002, 2020. DOI. 10.1088/1748-9326/ab97cc.

CHAZDON, R. L.; $\quad$ PERES, C. A.; $\quad$ DENT, D.; ShEIL, D.; LUGO, A. E.; LAMB, D.; STORK, N. E.; MILLER, S. E. The potential for species conservation in tropical secondary forests. Conservation Biology, v. 23, n. 6, p. 1406-1417, 2009. DOI. 10.1111/j.1523-1739.2009.01338.x.

CHOKKALINGAM, U.; JONG, W. D. Secondary forest: a working definition and typology. The International Forestry Review, Commonwealth Forestry Association, v. 3, n. 1, p. 19-26, 2001.

COE, M. T.; $\quad$ MARTHEWS, T. R.; $\quad$ COSTA, M. H.; $\quad$ GALBRAITH, D. R.; $\quad$ GREENGLASS, N. L.; IMBUZEIRO, H. M. A.; $\quad$ LEVINE, N. M.; MALHI, Y.; $\quad$ MOORCROFT, P. R.; $\quad$ MUZA, M. N.; POWELL, T. L.; SALESKA, S. R.; SOLORZANO, L. A.; WANG, J. Deforestation and climate feedbacks threaten the ecological integrity of south-southeastern Amazonia. Philosophical Transactions of the Royal Society B: Biological Sciences, v. 368, n. 1619, p. 1-9, 2013. DOI. 10.1098/rstb.2012.0155.

COMBER, A. J.; LAW, A. N. R.; LISHMAN, J. R. Application of knowledge for automated land cover change monitoring. International Journal of Remote Sensing, v. 25, n. 16, p. 3177-3192, 2004. DOI. 10.1080/01431160310001657795.

COPPIN, P. R.; JONCKHEERE, I.; NACKAERTS, K.; MUYS, B.; LAMBIN, E. Digital change detection methods in ecosystem monitoring: a review. International Journal of Remote Sensing, v. 25, n. 9, p. $1565-1596,2004$. DOI. 10.1080/0143116031000101675.

CORLETT, R. Tropical secondary forests. Progress in Physical Geography, v. 19, n. 2, p. 159-172, 1995. DOI. 10.1177/030913339501900201.

CORSINI, C. R. Análise multitemporal das mudanças de biomassa da vegetação secundária na Amazônia brasileira. 106 p. Tese (Doutorado) - Instituto Nacional de Pesquisas Espaciais (INPE), São José dos Campos, 2018. Disponível em: <http://urlib.net/rep/8JMKD3MGP3W34R/3R44PJP>. 
Acesso em: 12 maio 2019.

COSTA, F. A. Dinâmica agrária e balanço de carbono na Amazônia. Revista EconomiA, v. 10, n. 1, p. 117$151,2009$.

COSTA, F. A. Contributions of fallow lands in the Brazilian Amazon to $\mathrm{CO}_{2}$ balance, deforestation and the agrarian economy: inequalities among competing land use trajectories. Elementa Science of the Anthropocene, v. 4, 2016. DOI. 10.12952/journal.elementa.000133.

COUTINHO, A.; ALMEIDA, C.; VENTURIERI, A.; ESQUERDO, J.; SILVA, M. Uso e cobertura da terra nas áreas desflorestadas da Amazônia Legal: TerraClass 2008. 2013. Disponível em:< https://www.embrapa.br/busca-de-publicacoes/-/publicacao/971385/uso-e-cobertura-da-terra-nas-areasdesflorestadas-da-amazonia-legal-terraclass-2008>. Acesso em: 21 jul. 2020.

DI GREGORIO, A.; JANSEN, L. Land Cover Classification System. Food and Agriculture Organization of the United Nations: Rome, Italy, 2005.

EMPRESA BRASILEIRA DE PESQUISA AGROPECUÁRIA (EMBRAPA). Satélites de Monitoramento. 2018. Disponível em: <https://www.embrapa.br/satelites-de-monitoramento>. Acesso em: 18 dez. 2020.

EUROPEAN ENVIRONMENT AGENCY (EEA). Updated CLC illustrated nomenclature guidelines. 2019. Disponível em: <https://land.copernicus.eu/user-corner/technical-library/corine-land-covernomenclature-guidelines/docs/pdf/CLC2018_Nomenclature_illustrated_guide_20190510.pdf>. Acesso em 29 set. 2020.

EUROPEAN SPACE AGENCY (ESA). Sentinel Online. 2020. Disponível em: <https://sentinel.esa.int/web/sentinel/home>. Acesso em: $18 \mathrm{dez} .2020$.

FEARNSIDE, P. M. Environmental destruction in the Brazilian Amazon. In: HALL., A.; GOODMAN, D. (Eds.) The future of Amazonia. The Macmillan Press LTD: Houndmills, Basingstoke, Hampshire, London, 1990. p. 179-225.

FEARNSIDE, P. M. Deforestation in Brazilian Amazonia: history, rates, and consequences. Conservation Biology, v. 19, n. 3, p. 680-688, 2005. DOI. 10.1111/j.1523-1739.2005.00697.x.

FOLEY, J. A.; ASNER, G. P.; COSTA, M. H.; COE, M. T.; DEFRIES, R.; GIBBS, H. K.; HOWARD, E. A.; OLSON, S.; PATZ, J.; RAMANKUTTY, N.; SNYDER, P. Amazonia revealed: forest degradation and loss of ecosystem goods and services in the Amazon Basin. Frontiers in Ecology and the Environment, v. 5, n. 1, p. 25-32, 2007. DOI. 10.1890/1540-9295(2007)5[25:ARFDAL]2.0.CO;2.

FOODY, G. M. Assessing the accuracy of land cover change with imperfect ground reference data. Remote Sensing of Environment, v. 114, n. 10, p. 2271-2285, 2010. DOI. 10.1016/j.rse.2010.05.003.

FRANKLIN, S. E.; AHMED, O. S.; WUldER, M. A.; WHITE, J. C.; HERMOSILLA, T.; COOPS, N. C. Large area mapping of annual land cover dynamics using multitemporal change detection and classification of Landsat time series data. Canadian Journal of Remote Sensing, v. 41, n. 4, p. 293-314, 2015. DOI. 10.1080/07038992.2015.1089401.

FRANTZ, D. FORCE_Landsat+ Sentinel-2 analysis ready data and beyond. Remote Sensing, v. 11, n. 9, p. 1124, 2019. DOI. 10.3390/rs11091124.

FULLER, R.; SMITH, G.; DEVEREUX, B. The characterisation and measurement of land cover change through remote sensing: problems in operational applications? International Journal of Applied Earth Observation and Geoinformation, v. 4, n. 3, p. 243-253, 2003. DOI. 10.1016/S0303-2434(03)00004-7.

GILLANDERS, S. N.; COOPS, N. C.; WULDER, M. A.; GERGEL, S. E.; NELSON, T. Multitemporal remote sensing of landscape dynamics and pattern change: describing natural and anthropogenic trends. Progress in Physical Geography, v. 32, n. 5, p. 503-528, 2008. DOI. 10.1177/0309133308098363.

GÓMEZ, C.; WHITE, J. C.; WULDER, M. A. Optical remotely sensed time series data for land cover classification: a review. ISPRS Journal of Photogrammetry and Remote Sensing, v. 116, p. 55-72, 2016. DOI. 10.1016/j.isprsjprs.2016.03.008.

GONÇALVES, L. M.; FONTE, C. C., JULIO, E. N. B. S.; CAETANO, M. Evaluation of remote sensing images 
classifiers with uncertainty measures. In: DEVILLERS, R.; GOODCHILD, H. (Eds.). Spatial data quality from process to decisions. Boca Raton: CRC Press, 2009. p. 163-177.

GORELICK, N.; HANCHER, M.; DIXON, M.; ILYUSHCHENKO, S.; THAU, D.; MOORE, R. Google Earth Engine: planetary-scale geospatial analysis for everyone. Remote Sensing of Environment, v. 202, p. 18-27, 2017. DOI. 10.1016/j.rse.2017.06.031.

GRIFFITHS, P.; JAKIMOW, B.; HOSTERT, P. Reconstructing long term annual deforestation dynamics in Pará and Mato Grosso using the Landsat archive. Remote sensing of environment, v. 216, pp. 497-513, 2018. DOI. 10.1016/j.rse.2018.07.010.

GRIFFITHS, P.; VAN DER LINDEN, S.; KUEMMERLE, T.; HOSTERT, P. A pixel-based Landsat compositing algorithm for large area land cover mapping. IEEE Journal of Selected Topics in Applied Earth Observations and Remote Sensing, v. 6, n. 5, p. 2088-2101, 2013. DOI. 10.1109/JSTARS.2012.2228167.

GUHA, S.; RASTOGI, R.; SHIM, K. ROCK: A robust clustering algorithm for categorical attributes. Information Systems, v. 25, n. 5, p. 345-366, 2000. DOI. 10.1016/S0306-4379(00)00022-3.

HANSEN, M. C.; POTAPOV, P. V.; MOORE, R.; HANCHER, M.; TURUBANOVA, S. A.; TYUKAVINA, A.; THAU, D.; STEHMAN, S. V.; GOETZ, S. J.; LOVELAND, T. R.; KOMMAREDDY, A.; EGOROV, A.; CHINI, L; JUSTICE, C. O; TOWNSHEND, J. R. G. High-resolution global maps of 21st-century forest cover change. Science, v 342, n. 6160, p. 850-853, 2013. DOI. 10.1126/science.1244693

HERMOSILLA, T.; WULDER, M. A.; WHITE, J. C.; COOPS, N. C.; HOBART, G. W. Regional detection, characterization, and attribution of annual forest change from 1984 to 2012 using Landsat-derived time-series metrics. Remote Sensing of Environment, v. 170, p. 121-132, 2015. DOI. 10.1016/j.rse.2015.09.004

HERMOSILLA, T.; WULDER, M. A.; WHITE, J. C.; COOPS, N. C.; HOBART, G. W. Disturbance-informed annual land cover classification maps of Canada's forested ecosystems for a 29 -year Landsat time series. Canadian Journal of Remote Sensing, v. 44, n. 1, p. 67-87, 2018. DOI. 10.1080/07038992.2018.1437719.

HEROLD, M.; WOODCOCK, C.; DI GREGORIO, A.; MAYAUX, P.; BELWARD, A.; LATHAM, J.; SCHMULLIUS, C. A joint initiative for harmonization and validation of land cover datasets. IEEE Transactions on Geoscience and Remote Sensing, v. 44, n. 7, p. 1719-1727, 2006. DOI. 10.1109/TGRS.2006.871219.

HOLBEN, B. N. Characteristics of maximum-value composite images from temporal AVHRR data, International Journal of Remote Sensing, v. 7 n. 11, p. 1417-1434, $1986 . \quad$ DOI. $10.1080 / 01431168608948945$

HSU, C. C. Generalizing self-organizing map for categorical data. IEEE Transactions on Neural Networks, v. 17, n. 2, p. 294-304, 2006. DOI. 10.1109/TNN.2005.863415.

HUANG, S.; RAMIREZ, C.; KENNEDY, K.; MALLORY, J.; WANG, J.; CHU, C. Updating land cover automatically based on change detection using satellite images: case study of national forests in Southern California. GIScience \& Remote Sensing, v. 54, $\quad$ n. 4, p. 495-514, $2017 . \quad$ DOI. 10.1080/15481603.2017.1286727.

HUANG, Z. Extensions to the k-means algorithm for clustering large data sets with categorical values. Data mining and knowledge discovery, v. 2, n. 3, p. 283-304, 1998. DOI. 10.1023/A:1009769707641.

HUGHES, M. J.; KAYLOR, S. D.; HAYES, D. J. Patch-based forest change detection from Landsat time series. Forests, v. 8, n. 5, artigo 166, 2017, DOI. 10.3390/f8050166.

INSTITUTO BRASILEIRO DE GEOGRAFIA E ESTATÍSTICA (IBGE). Vocabulário básico de recursos naturais e meio ambiente. 2 ed. Rio de Janeiro, 2004. Disponível em: <https://ww2.ibge.gov.br/home/presidencia/noticias/vocabulario.pdf>. Acesso em: 6 mar. 2019.

INSTITUTO BRASILEIRO DE GEOGRAFIA E ESTATÍSTICA (IBGE). Manual Técnico de Uso da Terra. Rio de Janeiro, 2013. Disponível em: 〈https://biblioteca.ibge.gov.br/visualizacao/livros/liv81615.pdf〉. Acesso em: 29 ago. 2020. 
INSTITUTO BRASILEIRO DE GEOGRAFIA E ESTATÍSTICA (IBGE). Mudanças na cobertura e uso da terra 2000-2010-2012. Rio de Janeiro, 2015. Disponível em: <https://biblioteca.ibge.gov.br/visualizacao/livros/liv94724.pdf>. Acesso em: 21 jul. 2020.

INSTITUTO BRASILEIRO DE GEOGRAFIA E ESTATÍSTICA (IBGE). Monitoramento da cobertura e uso da terra do Brasil 2000-2010-2012-2014. Rio de Janeiro, 2017. Disponível em: <https://biblioteca.ibge.gov.br/visualizacao/livros/liv101469.pdf>. Acesso em: 21 jul. 2020.

INSTITUTO BRASILEIRO DE GEOGRAFIA E ESTATÍSTICA (IBGE). Monitoramento da cobertura e uso da terra do Brasil: 2016 - 2018. Rio de Janeiro, 2020. Disponível em: <https://biblioteca.ibge.gov.br/visualizacao/livros/liv101703.pdf>. Acesso em: 27 jul. 2020.

INSTITUTO HOMEM E MEIO AMBIENTE DA AMAZÔNIA (IMAZON). Monitoramento da Amazônia. 2020. Disponível em:< https://imazon.org.br/>. Acesso em: 02 out. 2020.

INSTITUTO NACIONAL DE PESQUISAS ESPACIAIS (INPE). Nota Técnica - Correção da máscara do PRODES. $2017 . \quad$ Disponível em: <http://www.obt.inpe.br/OBT/assuntos/programas/amazonia/prodes/pdfs/nt_deslocamentomascara.pdf>. Acesso em: 02 out. 2020.

INSTITUTO NACIONAL DE PESQUISAS ESPACIAIS (INPE). Satélites. 2020a. Disponível em: <http://www.dgi.inpe.br/documentacao/satelites>. Acesso em: 18 dez. 2020.

INSTITUTO NACIONAL DE PESQUISAS ESPACIAIS (INPE). CBERS: Satélite Sino-Brasileiro de Recursos Terrestres. 2020b. Disponível em: < http://www.cbers.inpe.br/>. Acesso em: 18 dez. 2020.

INSTITUTO NACIONAL DE PESQUISAS ESPACIAIS (INPE). Monitoramento do Desmatamento da Floresta Amazônica Brasileira por Satélite. 2020c. Disponível em: <http://www.obt.inpe.br/OBT/assuntos/programas/amazonia/prodes>. Acesso em: 02 out. 2020.

INSTITUTO NACIONAL DE PESQUISAS ESPACIAIS (INPE). DETER e DETER intenso. 2020d. Disponível em: <http://www.obt.inpe.br/OBT/assuntos/programas/amazonia/deter>. Acesso em: 02 out. 2020.

JAKIMOW, B.; VAN DER LINDEN, S.; THIEL, F.; FRANTZ, D.; HOSTERT, P. Visualizing and labeling dense multi-sensor earth observation time series: The EO Time Series Viewer. Environmental Modelling \& Software, v. 125, artigo 104631, 2020. DOI. 10.1016/j.envsoft.2020.104631.

JAKOVAC, C. C.; DUTRIEUX, L. P.; SITI, L.; PEÑA-CLAROS, M.; BONGERS, F. Spatial and temporal dynamics of shifting cultivation in the middle-Amazonas river: Expansion and intensification. PloS one, v. 12, n. 7, e0181092, 2017. DOI. 10.1371/journal.pone.0181092.

KAMMESHEIDT, L. Perspectives on secondary forest management in Tropical Humid Lowland America. Ambio, v. 31, n. 3, p. 243-250, 2002. DOI. 10.1579/0044-7447-31.3.243.

KENNEDY, R. E., YANG, Z;; COHEN, W. B. Detecting trends in forest disturbance and recovery using yearly Landsat time series: 1. LandTrendr - Temporal segmentation algorithms. Remote Sensing of Environment, v. 114, n. 12, p. 2897-2910, 2010. DOI. 10.1016/j.rse.2010.07.008.

LIU, H.; ZHOU, Q. Accuracy analysis of remote sensing change detection by rule-based rationality evaluation with post-classification comparison. International Journal of Remote Sensing, v. 25, n. 5, p. 1037-1050, 2004. DOI. 10.1080/0143116031000150004

LU, D.; LI, G.; MORAN, E. Current situation and needs of change detection techniques. International Journal of Image and Data Fusion, v. 5, n. 1, p. 13-38, 2014. DOI. 10.1080/19479832.2013.868372.

LU, D.; MAUSEL, P.; BRONDÍZIO, E.; MORAN, E. Change detection techniques. International Journal of Remote Sensing, v. 25, n. 12, p. 2365-2401, 2004. DOI. 10.1080/0143116031000139863.

MACIEL, A. M.; CÂMARA, G.; VINHAS, L.; PICOLI, M. C. A.; BEGOTTI, R. A.; ASSIS, L. F. F. G. D. A spatiotemporal calculus for reasoning about land-use trajectories. International Journal of Geographical Information Science, v. 33, n. 1, p. 176-192, 2019. DOI. 10.1080/13658816.2018.1520235.

MAPBIOMAS. MapBiomas General "Handbook": Algorithm Theoretical Basis Document (ATBD) - 
Collection 2020. D, Disponível em: <https://mapbiomas-brsite.s3.amazonaws.com/ATBD_Collection_5_v1.pdf>. Acesso em: 30 set. 2020.

MAURANO, L. E. P.; ESCADA, M. I. S. Comparação dos dados produzidos pelo PRODES versus dados do MapBiomas para o bioma Amazônia. In: SIMPÓSIO BRASILEIRO DE SENSORIAMENTO REMOTO, 19. (SBSR), 2019, Santos. Anais... São José dos Campos: INPE, 2019. p. 735-738. Disponível em: <http://urlib.net/rep/8JMKD3MGP6W34M/3U24J5L>. Acesso em: 01 jul. 2020.

MAUS, V.; CÂMARA, G.; CARTAXO, R.; SANCHEZ, A.; RAMOS, F. M.; QUEIROZ, G. R. A timeweighted dynamic time warping method for land-use and land-cover mapping. IEEE Journal of Selected Topics in Applied Earth Observations and Remote Sensing, v. 9, n. 8, p. 3729-3739, 2016. DOI. 10.1109/JSTARS.2016.2517118.

MCCONNELL, W.; MORAN, E. Meeting in the Middle: The Challenge of Meso-Level Integration, LUCC Report Series No. 5, Land Utilization Coordinating Council: Rome, Italy, 2001. Disponível em: <http://www.comap.ca/kmland/getdocument.php?F=310836083_4c7195ce74a518.86955292>. Acesso em: 30 jul. 2020.

MELLO, A. Y. I.; ALVES, D. S. Secondary vegetation dynamics in the Brazilian Amazon based on Thematic Mapper imagery. Remote Sensing Letters, v. 2, n. 3, p. 189-194, 2011. DOI. 10.1080/01431161.2010.506206.

MENA, C. F. Trajectories of land-use and land-cover in the northern Ecuadorian Amazon. Photogrammetric Engineering \& Remote Sensing, v. 74, n. 6, p. 737-751, 2008. DOI. 10.14358/PERS.74.6.737.

MERTENS, B.; LAMBIN, E. Land cover change trajectories in southern Cameroon. Annals of the Association of American Geographers, v. 90, n. 3, p. 467-494, 2000. DOI. 10.1111/0004-5608.00205.

MEYFROIDT, P.; LAMBIN, E. F. Global forest transition: prospects for an end to deforestation. Annual Review of Environment and Resources, v. 36, n. 1, p. 343-371, 2011. DOI. 10.1146/annurev-environ090710-143732.

MORAN, E. F.; BRONDIZIO, E. S.; TUCKER, J. M.; SILVA-FORSBERG, M. C.; MCCRACKEN, S.; FALESI, I. Effects of soil fertility and land-use on forest succession in Amazonia. Forest Ecology and Management, v. 139, n. 1, p. 93-108, 2000. DOI. 10.1016/S0378-1127(99)00337-0.

MÜLLER, H.; RUFIN, P.; GRIFFITHS, P.; HISSA, L. D. B. V.; HOSTERT, P. Beyond deforestation: Differences in long-term regrowth dynamics across land use regimes in southern Amazonia. Remote Sensing of Environment, v. 186, p. 652-662, 2016. DOI. 10.1016/j.rse.2016.09.012.

MÜllER-HANSEN, F.; $\quad$ CARDOSO, M. F.; $\quad$ DALlA-NORA, E. L.; $\quad$ DONGES, J. F.; $\quad$ HEITZIG, J.; KURTHS, J.; THONICKE, K. A matrix clustering method to explore patterns of land-cover transitions in satellite-derived maps of the Brazilian Amazon. Nonlinear Processes in Geophysics, v. 24, n. 1, p. 113-123, 2017. DOI. 10.5194/npg-24-113-2017.

NATIONAL AERONAUTICS AND SPACE ADMINISTRATION (NASA). MODIS: Moderate Resolution Imaging Spectroradiometer. 2020a. Disponível em: <https://modis.gsfc.nasa.gov/>. Acesso em 18 dez. 2020.

NATIONAL AERONAUTICS AND SPACE ADMINISTRATION (NASA). Terra: the EOS Flagship. 2020b. Disponível em: <https://terra.nasa.gov/>. Acesso em: 18 dez. 2020.

NEVES, A. K.; KÖRTING, T. S.; FONSECA, L. M. G.; ESCADA, M. I. S. Assessment of TerraClass and MapBiomas data on legend and map agreement for the Brazilian Amazon biome. Acta Amazonica, v. 50, n. 2, p 170-182, 2020. DOI. 10.1590/1809-4392201900981.

NUNES, S.; OLIVEIRA JR, L.; SIQUEIRA, J.; MORTON, D. C.; SOUZA JR, C. M. Unmasking secondary vegetation dynamics in the Brazilian Amazon. Environmental Research Letters, v. 15, n. 3, artigo 034057, 2020. DOI. 10.1088/1748-9326/ab76db.

OLOFSSON, P.; FOODY, G. M.; HEROLD, M.; STEHMAN, S. V.; WOODCOCK, C. E.; WULDER, M. A. Good practices for estimating area and assessing accuracy of land change. Remote Sensing of 
Environment, v. 148, p. 42-57, 2014. DOI. 10.1016/j.rse.2014.02.015.

PANTALEÃO, E.; DUTRA, L. V.; SANDRI, S. A. Scenario analysis for image classification using multiobjective optimization. InfoComp, v. 1, n. 3-4, p. 15-22, 2012.

PARÁ. Instrução Normativa $n^{\circ} 08$, de 28 de outubro de 2015. Define procedimentos administrativos para a realização de limpeza e autorização de supressão, a serem realizadas nas áreas de vegetação secundária em estágio inicial de regeneração, localizadas fora da Reserva Legal e da Área de Preservação Permanente - APP dos imóveis rurais, no âmbito do Estado do Pará, e dá outras providências. DOE 33.003, Belém, PA, 03 nov. 2015. p. 31-33.

PARADELLA, W.; SANTOS, A.; VENEZIANI, P.; CUNHA, E. Radares imageadores nas geociências. Revista Brasileira de Cartografia, v. 1, n. 57, p. 56-62, 2005.

PEREIRA, L. O.; FREITAS, C. C.; SANT'ANNA, S. J. S.; REIS, M. S. Evaluation of Optical and Radar Images Integration Methods for LULC Classification in Amazon Region. IEEE Journal of Selected Topics in Applied Earth Observations and Remote Sensing, v. 11, n. 9, p. 3062-3074, 2018. DOI. 10.1109/JSTARS.2018.2853647.

PERZ, S. G.; SKOLE, D. L. Secondary forest expansion in the Brazilian Amazon and the refinement of Forest Transition Theory. Society \& Natural Resources, v. 16, n. 4, p.277-294, 2003. DOI. 10.1080/08941920390178856.

PINHEIRO, T. F.; ESCADA, M. I. S.; VALERIANO, D.; HOSTERT, P.; GOLLNOW, F.; MÜLLER, H. Forest degradation associated with logging frontier expansion in the Amazon: the BR-163 region in southwestern Pará, Brazil. Earth Interactions, v. 20, n. 17, p. 1-26, 2016. DOI. 10.1175/EI-D-15-0016.1.

POWELL, R.; MATZKE, N.; SOUZA, C.; CLARK, M.; NUMATA, I.; HESS, L.; ROBERTS, D. Sources of error in accuracy assessment of thematic land-cover maps in the Brazilian Amazon. Remote Sensing of Environment, v. 90, n. 2, p. 221-234, 2004. DOI. 10.1016/j.rse.2003.12.007.

PUTZ, F. E.; REDFORD, K. H. The importance of defining 'forest': Tropical forest degradation, deforestation, long-term phase shifts, and further transitions. Biotropica, v. 42, n. 1, p. 10-20, 2010. DOI. 10.1111/j.1744-7429.2009.00567.x.

RAMANKUTTY, N.; GIBBS, H. K.; ACHARD, F.; DEFRIES, R.; FOLEY, J. A.; HOUGHTON, R. A. Challenges to estimating carbon emissions from tropical deforestation. Global Change Biology, v. 13, n. 1, p. 51-66, 2007. DOI. 10.1111/j.1365-2486.2006.01272.x.

REIS, M. S.; DUTRA, L. V.; ESCADA, M. I. S.; SANT'ANNA, S. J. S Avoiding invalid transitions in land cover trajectory classification with a Compound Maximum a Posteriori approach. IEEE Access, v. 8, p. 98787-98799, 2020. DOI. 10.1109/ACCESS.2020.2997019.

REIS, M. S.; DUTRA, L. V.; SANT'ANNA, S. J. S.; ESCADA, M. I. S. Análise das incertezas envolvidas em classificação multi-legendas da cobertura da terra com suporte de simulação Monte Carlo. Revista Brasileira de Cartografia, v. 69, n. 9, p. 1725-1741, 2017.

REIS, M. S.; $\quad$ ESCADA, M. I. S.; DUTRA, L. V.; SANT’ANNA, S. J. S.; VOGT, N. D. Towards a reproducible LULC hierarchical class legend for use in the southwest of Pará State, Brazil: a comparison with remote sensing data-driven hierarchies. Land, v. 7, n. 2, artigo 65, 2018. DOI. 10.3390/land7020065.

RICHARDS, J. A.; JIA, X. Remote sensing digital image analysis: an introduction. 4. ed. Germany: Springer, 2006.

RUFIN, P.; MÜLlER, H.; PFLUGMACHER, D.; HOSTERT, P. Land use intensity trajectories on Amazonian pastures derived from Landsat time series. International Journal of Applied Earth Observation and Geoinformation, v. 41, p. 1-10, 2015. DOI. 10.1016/j.jag.2015.04.010.

SALOMÃO, R. P.; VIEIRA, I. C. G.; BRIENZA JÚNIOR, S.; AMARAL, D. D.; SANTANA, A. C. Sistema capoeira classe: uma proposta de sistema de classificação de estágios sucessionais de florestas secundárias para o estado do Pará. Boletim do Museu Paraense Emílio Goeldi. Ciências Naturais, v. 7, n. 3, p. 297-317, 2012. 
SANCHEZ, A. H.; PICOLI, M. C. A.; CÂMARA, G.; ANDRADE, P. R.; CHAVES, M. E. D.; LECHLER, S.; SOARES, A. R.; MARUJO, R. F. B.; SIMÕES, R. E. O. ; FERREIRA, K. R.; QUEIROZ, G. R. Comparison of Cloud Cover Detection Algorithms on Sentinel-2 Images of the Amazon Tropical Forest. Remote Sensing, v. 12, n. 08, artigo 1284, 2020. DOI. 10.3390/rs12081284.

SEXTON, J. O.; URBAN, D. L.; DONOHUE, M. J.; SONG, C. Long-term land cover dynamics by multitemporal classification across the Landsat-5 record. Remote Sensing of Environment, v. 128, p. 246258, 2013. DOI. 10.1016/j.rse.2012.10.010.

SHIMABUKURO, Y. E.; SMITH, J. A. Fraction images derived from Landsat TM and MSS data for monitoring reforested areas. Canadian Journal of Remote Sensing, v. 21, n. 1, p. 67-74, 1995. DOI. 10.1080/07038992.1995.10874599.

SHIMADA, M.; ITOH, T.; MOTOOKA, T.; WATANABE, M.; SHIRAISHI, T.; THAPA, R.; LUCAS, R. New global forest/non-forest maps from ALOS PALSAR data (2007-2010). Remote Sensing of Environment, v. 155, p. 13-31, 2014. DOI. 10.1016/j.rse.2014.04.014

SILVA JUNIOR, C. H. L.; HEINRICH, V. H. A.; FREIRE, A. T. G.; BROGGIO, I. S.; ROSAN, T. M.; DOBLAS, J.; ANDERSON, L. O.; ROUSSEAU, G. X.; SHIMABUKURO, Y. E.; SILVA, C. A.; HOUSE, J. I.; ARAGÃO, L.E.O.C. Benchmark maps of 33 years of secondary forest age for Brazil. Scientific Data, v. 7, n. 1, p. 269-269, 2020. DOI. 10.1038/s41597-020-00600-4.

SOUZA, C. M.; SHIMBO, J. Z.; ROSA, M. R.; PARENTE, L. L.; ALENCAR, A. A.; RUDORFF, B. F. T.; HASENACK, H.; MATSUMOTO, M.; FERREIRA, L. G.; SOUZA-FILHO, P. W. M.; OLIVEIRA, S. W.; ROCHA, W. F.; FONSECA, A. V.; MARQUES, C. B.; DINIZ, C. G.; COSTA, D.; MONTEIRO, D.; ROSA, E. R.; VÉLEZ-MARTIN, E.; WEBER, E. J.; LENTI, F. E. B.; PATERNOST, F. F.; PAREYN, F. G. C.; SIQUEIRA, J. V.; VIERA, J. L.; FERREIRA NETO, L. C.; SARAIVA, M. M.; SALES, M. H.; SALGADO, M. P. G.; VASCONCELOS, R.; GALANO, S.; MESQUITA, V. V.; AZEVEDO, T. Reconstructing Three Decades of Land Use and Land Cover Changes in Brazilian Biomes with Landsat Archive and Earth Engine. Remote Sensing, v. 12, n. 17, artigo 2735, 2020. DOI. $10.3390 / \mathrm{rs} 12172735$.

STEININGER, M. Secondary forest structure and biomass following short and extended land-use in central and southern Amazonia. Journal of tropical Ecology, v. 16, p. 689-708, 2000.

TEWKESBURY, A. P.; COMBER, A. J.; TATE, N. J.; LAMB, A.; FISHER, P. F. A critical synthesis of remotely sensed optical image change detection techniques. Remote Sensing of Environment, v. 160, p. 1-14, 2015. DOI. 10.1016/j.rse.2015.01.006.

TURNER, B.; MEYER, W. Global land-use and land-cover change: an overview. In: MEYER, W.; TURNER, B. (Ed.). Changes in land use and land cover: a global perspective. Cambridge: Cambridge University Press, 1994.

UNITED STATES GEOLOGICAL SURVEY (USGS). USGS EROS Archive - Products Overview. 2020. Disponível em: <https://www.usgs.gov/centers/eros/science/usgs-eros-archive-products-overview?qtscience_center_objects=0\#qt-science_center_objects $>$. Acesso em: 18 dez. 2020.

VAN ROESSEL, J. W.; GODOY, R. C. SLAR mosaics for project RADAM. Photogrammetric Engineering, v. 40, p. 583-595, 1974.

VERBESSELT, J.; HYNDMAN, R.; NEWNHAM, G.; CULVENOR, D. Detecting trend and seasonal changes in satellite image time series. Remote Sensing of Environment, v. 114, n. 1, p. 106-115, 2010. DOI. 10.1016/j.rse.2009.08.014.

VIEIRA, I. C. G.; GARDNER, T.; FERREIRA, J.; LEES, A. C.; BARLOW, J. Challenges of governing second-growth forests: A case study from the Brazilian Amazonian State of Pará. Forests, v. 5, n. 7, p. 1737-1752, 2014. DOI. 10.3390/f5071737.

WANDELLI, E. V.; FEARNSIDE, P. M. Secondary vegetation in central Amazonia: Land-use history effects on aboveground biomass. Forest Ecology and Management, v. 347, p. 140-148, 2015. DOI. 10.1016/j.foreco.2015.03.020. 
WANG, Y.; ZIV, G.; ADAMI, M.; ALMEIDA, C. A.; ANTUNES, J. F. G.; COUTINHO, A. C.; ESQUERDO, J. C. D. M.; GOMES, A. R.; GALBRAITH, D. Upturn in secondary forest clearing buffers primary forest loss in the Brazilian Amazon. Nature Sustainability, v. 3, n. 4, p. 290-295, 2020. DOI. 10.1038/s41893-019-0470-4.

WHITE, J. C.; WULDER, M. A.; HOBART, G. W.; LUTHER, J. E.; HERMOSILLA, T.; GRIFFITHS, P., COOPS, N. C.; HALL, R. J.; HOSTERT, P.; DYK, A,; GUINDON, L. Pixel-based image compositing for large-area dense time series applications and science. Canadian Journal of Remote Sensing, v. 40, n. 3, p. 192-212, 2014. DOI. 10.1080/07038992.2014.945827

WULDER, M. A.; COOPS, N. C.; ROY, D. P.; WHITE, J. C.; HERMOSILLA, T. Land cover 2.0. International Journal of Remote Sensing, v. 39, n. 12, p. 4254-4284, 2018. DOI. 10.1080/01431161.2018.1452075.

XIAN, G.; HOMER, C. Updating the 2001 National Land Cover Database impervious surface products to 2006 using Landsat imagery change detection methods. Remote Sensing of Environment, v. 114, n. 8, p. 1676-1686, 2010. DOI. 10.1016/j.rse.2010.02.018.

YUAN, F.; SAWAYA, K. E.; LOEFFELHOLZ, B. C.; BAUER, M. E. Land cover classification and change analysis of the Twin Cities (Minnesota) Metropolitan Area by multitemporal Landsat remote sensing. Remote Sensing of Environment, v. 98, n. 2, p. 317-328, 2005. DOI. 10.1016/j.rse.2005.08.006.

ZHU, Z. Change detection using landsat time series: A review of frequencies, preprocessing, algorithms, and applications. ISPRS Journal of Photogrammetry and Remote Sensing, v. 130, p. 370-384, 2017. DOI. 10.1016/j.isprsjprs.2017.06.013.

ZHU, Z.; FU, Y.; WOODCOCK, C. E.; OLOFSSON, P.; VOGELMANN, J. E.; HOLDEN, C.; WANG, M.; DAI, S.; YU, Y. Including land cover change in analysis of greenness trends using all available Landsat 5, 7, and 8 images: a case study from Guangzhou, China (2000-2014). Remote Sensing of Environment, v. 185, p. 243-257, 2016. DOI. 10.1016/j.rse.2016.03.036.

ZHU, Z.; WOODCOCK, C.E. Continuous change detection and classification of land cover using all available Landsat data. Remote Sensing of Environment, v. 144, p. 152-171, 2014. DOI. 10.1016/j.rse.2014.01.011.

ZOUNGRANA, B. J.-B.; CONRAD, C.; AMEKUDZI, L. K.; THIEL, M.; DA, E. D.; FORKUOR, G.; LÖW, F. Multi-temporal Landsat images and ancillary data for land use/cover change (LULCC) detection in the Southwest of Burkina Faso, West Africa. Remote Sensing, v. 7, n. 9, p. 12076-12102, 2015. DOI. 10.3390/rs70912076.

\section{Biografia dos autores}

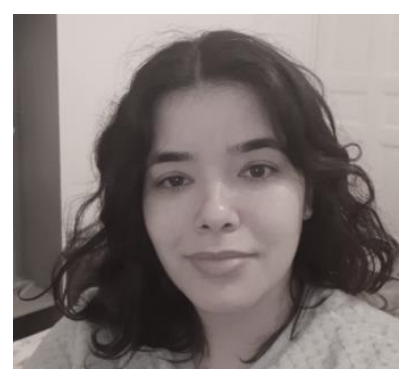

\footnotetext{
Mariane Souza Reis se formou em Engenharia Ambiental pela Pontifícia Universidade Católica de Campinas, Campinas, 2011. Concluiu o mestrado em Sensoriamento Remoto pelo Instituto Nacional de Pesquisas Espaciais (INPE), São José dos Campos, em 2014. Atualmente está matriculada no curso de Doutorado em Ciência do Sistema Terrestre. De 2014 a 2016, trabalhou no INPE com monitoramento da cobertura da terra, em atividades de campo na Amazônia, e com o desenvolvimento de algoritmos de processamento de imagens. Seus interesses de pesquisa incluem análises de uso e cobertura da terra e técnicas de processamento de imagens.
} 

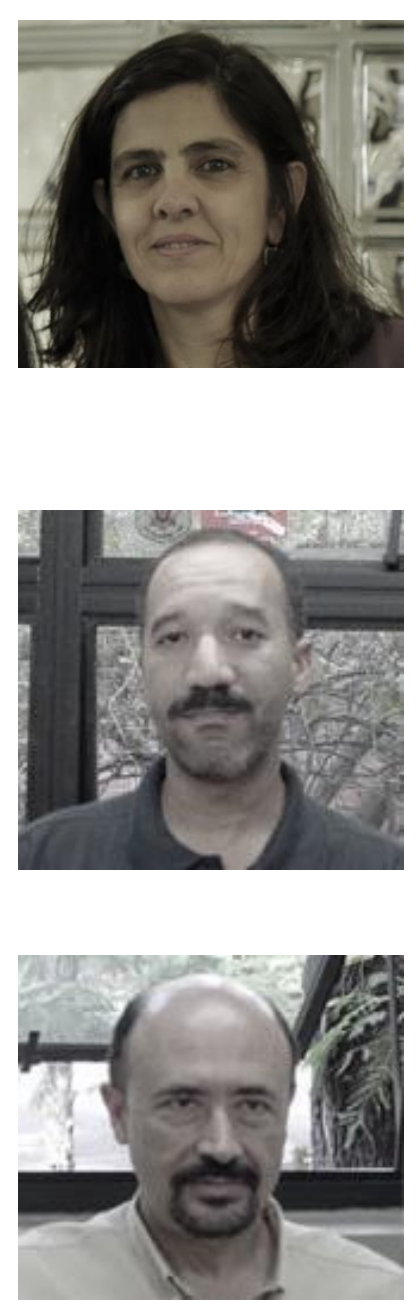

Maria Isabel Sobral Escada se formou em ecologia pela Universidade Estadual Paulista 'Júlio de Mesquita Filho', em 1987. Concluiu o mestrado e o doutorado em Sensoriamento Remoto no INPE, respectivamente em 1992 e 2003. Possui experiência com tecnologias de sensoriamento remoto e técnicas de análise especial aplicadas a estudos da paisagem, pela análise de padrões de mudanças de uso e cobertura da terra na Amazônia. Atualmente, é pesquisadora sênior na Divisão de Observação da Terra e Geoinformática e professora nos programas de pós-graduação em Ciência do Sistema Terrestre e em Sensoriamento Remoto do INPE.

Sidnei João Siqueira Sant'anna se formou em engenharia elétrica e eletrônica pela Universidade Federal do Rio de Janeiro, Rio de Janeiro, Brasil, em 1993. Concluiu o mestrado em Sensoriamento Remoto no INPE em 1995, e o doutorado em Engenharia Eletrônica e Computação pelo Instituto Tecnológico da Aeronáutica (ITA), São José dos Campos, em 2009. Atualmente trabalha como pesquisador no INPE. Seus interesses de pesquisa incluem análise de imagens e técnicas de processamento aplicadas a sensoriamento remote, filtragem de dados SAR, métodos estatísticos, robustez, e assuntos relacionados.

Luciano Vieira Dutra possui graduação em Engenharia Eletrônica pelo ITA (1976), mestrado em Computação Aplicada pelo INPE (1981), e doutorado em Computação Aplicada pelo INPE (1989). Trabalha no INPE desde 1977, em diversos projetos de sensoriamento remoto de larga escala, nacionais e internacionais. Atualmente é professor dos programas de Computação Aplicada e Sensoriamento Remoto, ambos no INPE, em que leciona os cursos de Processamento de Imagem e Sinal, Reconhecimento de Padrões, e Processamento de Imagens de Radar. 